\title{
SURFACES EXPANDING BY THE INVERSE GAUSS CURVATURE FLOW
}

\author{
OLIVER C. SCHNÜRER
}

\begin{abstract}
We show that strictly convex surfaces expanding by the inverse Gauß curvature flow converge to infinity in finite time. After appropriate rescaling, they converge to spheres. We describe the algorithm to find our main test function.
\end{abstract}

\section{Contents}

1. Introduction 1

2. Notation 3

3. A Monotone Quantity 4

4. Convergence to Infinity 8

5. Convergence to a Sphere 11

6. Smooth Convergence to a Sphere 12

7. Improved Convergence Rate 13

8. Finding Monotone Quantities 17

9. Computing Evolution Equations 17

10. Other Normal Velocities 19

11. Convergence Rate 24

References $\quad 24$

\section{INTRODUCTION}

We consider a family of closed strictly convex surfaces $M_{t}$ in $\mathbb{R}^{3}$ that expand by the inverse Gauß curvature flow

$$
\frac{d}{d t} X=\frac{1}{K} \nu
$$

This is a parabolic flow equation. We obtain a solution on a maximal time interval $[0, T), 0<T<\infty$. For $t \uparrow T$, the surfaces converge to infinity. After appropriate rescaling, they converge to a round sphere. We say that the surfaces $M_{t}$ converge to "round spheres at infinity". The key step in the proof, Theorem 3.1, is to show that

$$
\max _{M_{t}}\left(\frac{\left(\lambda_{1}-\lambda_{2}\right)^{2}}{\lambda_{1}^{2} \lambda_{2}^{2}}\right)
$$

is non-increasing in time.

Date: December 2004, revised February 2006.

2000 Mathematics Subject Classification. Primary 53C44, 65K05; Secondary 35B40.

The author is a member of SFB 647/B3 "Raum - Zeit - Materie". 


\begin{tabular}{|c||c|}
\hline$-\frac{1}{K}$ & $\frac{\left(\lambda_{1}-\lambda_{2}\right)^{2}}{\lambda_{1}^{2} \lambda_{2}^{2}}$ \\
\hline$-\frac{H^{2}}{K^{2}}$ & $\frac{\left(\lambda_{1}-\lambda_{2}\right)^{2}}{\left(\lambda_{1}+\lambda_{2}\right) \lambda_{1} \lambda_{2}}$ \\
\hline$-\frac{|A|^{2}}{K^{2}}$ & $\frac{\left(\lambda_{1}-\lambda_{2}\right)^{2}}{\left(\lambda_{1}+\lambda_{2}\right) \lambda_{1} \lambda_{2}}$ \\
\hline$-\frac{H^{3}}{K^{3}}$ & $\frac{\left(\lambda_{1}+\lambda_{2}\right)^{6}\left(\lambda_{1}-\lambda_{2}\right)^{2}}{\left(\lambda_{1}^{2}+\lambda_{2}^{2}\right)\left(\lambda_{1}^{2}+\lambda_{1} \lambda_{2}+\lambda_{2}^{2}\right) \lambda_{1}^{3} \lambda_{2}^{3}}$ \\
\hline
\end{tabular}

TABLE 1. Monotone quantities

Here, we used standard notation as explained in Section 2.

Our main theorem is

Theorem 1.1. For any smooth closed strictly convex surface $M$ in $\mathbb{R}^{3}$, there exists a smooth family of surfaces $M_{t}, t \in[0, T)$, solving (1.1) with $M_{0}=M$. For $t \uparrow T$, the surfaces $M_{t}$ converge to infinity. The rescaled surfaces $M_{t} \cdot(T-t)$ converge smoothly to the unit sphere $\mathbb{S}^{2}$.

We will also consider other normal velocities for which similar results hold. Therefore, we have to find quantities like (1.2) that are monotone during the flow and vanish precisely for spheres. In general, this is a complicated issue. In order to find these test quantities, we used an algorithm that checks, based on randomized tests, whether possible candidates fulfill certain inequalities. These inequalities guarantee especially that we can apply the maximum principle to prove monotonicity. We used that algorithm only to propose useful quantities. The presented proof does not depend on it. So far, all candidates proposed by the corresponding program turned out to be appropriate for proving convergence to round spheres at infinity. In Table 1 , we have collected some normal velocities $F$ ( $1^{\text {st }}$ column) and quantities $w\left(2^{\text {nd }}\right.$ column $)$ such that $\max _{M_{t}} w$ is non-increasing in time for surfaces expanding with normal velocity $F$

$$
\frac{d}{d t} X=-F \nu
$$

It is common to use positive functions $F$ for contracting surfaces. Thus the negative sign corresponds to the fact that these surfaces expand. In each case, we obtain convergence to round spheres at infinity for smooth closed strictly convex initial surfaces $M_{0}$.

There are many papers concerning convex hypersurfaces contracting to "round points", i. e. the surfaces converge to a point, and, after appropriate rescaling, to a sphere, especially for normal velocities homogeneous of degree one in the principal curvatures, see e.g. [14] for motion by mean curvature. For normal velocities of higher homogeneity, strictly convex hypersurfaces converge to a point [28] and to a round point, if they are appropriately pinched initially, e. g. [2] and [24] for surfaces. Convex surfaces contracting with normal velocities homogeneous of degree 
larger than one in the principal curvatures converge to round points without initial pinching assumption, see [5] for the Gauß curvature flow and [21] for other flow equations and strictly convex surfaces.

Expanding flows of homogeneity minus one, i.e. flows of the form $\frac{d}{d t} X=-F \nu$ with $F$ positive homogeneous of degree minus one, were studied by Claus Gerhardt and John Urbas $[11,29,30]$. They obtain convergence to round spheres at infinity. These results extend to negative homogeneities larger than minus one. Note that solutions exist for $t \in[0, \infty)$. There is a representation formula for solutions to the inverse harmonic mean curvature flow of Knut Smoczyk [26]. Gerhard Huisken and Tom Ilmanen used the inverse mean curvature flow to prove the Penrose inequality [15]. This was extended in $[16,25]$. There are also inhomogeneous flows for which solutions converge to round spheres as $t \uparrow \infty[6,9,10,18]$.

Our paper concerns the expansion of surfaces by the inverse Gauß curvature flow. This flow is homogeneous of degree minus two in the principal curvatures. Solutions tend to round points at infinity in finite time. We want to stress that we don't have to assume any pinching condition for the initial surface.

The rest of this paper is organized as follows. In Section 2, we explain our notation. Section 3 concerns the key step, Theorem 3.1, the proof of the monotonicity of our test function during the flow. We prove convergence to infinity for appropriate points on the surfaces in Section 4. In Section 5, we obtain that the surfaces converge to infinity and, after appropriate rescaling, to a round sphere in Hausdorff distance. We improve this in Section 6 and get smooth convergence to a round sphere after rescaling. This finishes the proof of our main theorem. A further improvement of this result is contained in Section 7. There, we show that our surfaces converge in Hausdorff distance to a family of spheres expanding by inverse Gauß curvature flow.

In Sections 8 and 9, we describe the computational aspects of our flow equation. We describe the algorithm that we used to find our monotone quantities. In this paper, we compute complicated evolution equations. We describe, how this can be done with a computer algebra program.

We also get convergence to round spheres at infinity for other flow equations. These are considered in Section 10. Finally, we derive the optimal expected convergence rate in Section 11.

The author wants to thank Klaus Ecker at the Free University Berlin for discussions and support, especially for telling us about Aleksandrov reflection for parabolic equations. We also want to thank Jörg Härterich, Gerhard Huisken, and Felix Otto for discussions concerning the optimal convergence rate.

\section{Notation}

We use $X=X(x, t)$ to denote the embedding vector of a manifold $M$ into $\mathbb{R}^{3}$ and $\frac{d}{d t} X=\dot{X}$ for its total time derivative. Set $M_{t}:=X(M, t) \subset \mathbb{R}^{3}$. We choose $\nu$ to be the outer unit normal vector of $M_{t}$. The embedding induces a metric $\left(g_{i j}\right)$ and a second fundamental form $\left(h_{i j}\right)$. We use the Einstein summation convention. Indices are raised and lowered with respect to the metric or its inverse $\left(g^{i j}\right)$. The inverse of the second fundamental form is denoted by $\left(\tilde{h}^{i j}\right)$. The principal curvatures $\lambda_{1}, \lambda_{2}$ are the eigenvalues of the second fundamental form with respect to the metric. A surface is called strictly convex, if all principal curvatures are strictly positive. We will assume this throughout the paper. 
Symmetric functions of the principal curvatures are well-defined, we will use the mean curvature $H=\lambda_{1}+\lambda_{2}$, the square of the norm of the second fundamental form $|A|^{2}=\lambda_{1}^{2}+\lambda_{2}^{2}, \operatorname{tr} A^{k}=\lambda_{1}^{k}+\lambda_{2}^{k}$, and the Gauß curvature $K=\lambda_{1} \lambda_{2}$. We write indices, preceded by semi-colons, e.g. $h_{i j ; k}$, to indicate covariant differentiation with respect to the induced metric. It is often convenient to choose coordinate systems such that, at a fixed point, the metric tensor equals the Kronecker delta, $g_{i j}=\delta_{i j}$, and $\left(h_{i j}\right)$ is diagonal, $\left(h_{i j}\right)=\operatorname{diag}\left(\lambda_{1}, \lambda_{2}\right)$, e.g.

$$
\sum \lambda_{k} h_{i j ; k}^{2}=\sum_{i, j, k=1}^{2} \lambda_{k} h_{i j ; k}^{2}=h^{k l} h_{j ; k}^{i} h_{i ; l}^{j}=h_{r s} h_{i j ; k} h_{a b ; l} g^{i a} g^{j b} g^{r k} g^{s l} .
$$

Whenever we use this notation, we will also assume that we have fixed such a coordinate system. We will only use Euclidean coordinate systems for $\mathbb{R}^{3}$ so that $h_{i j ; k}$ is symmetric according to the Codazzi equations.

A normal velocity $F$ can be considered as a function of $\left(\lambda_{1}, \lambda_{2}\right)$ or $\left(h_{i j}, g_{i j}\right)$. We set $F^{i j}=\frac{\partial F}{\partial h_{i j}}, F^{i j, k l}=\frac{\partial^{2} F}{\partial h_{i j} \partial h_{k l}}$. Note that in coordinate systems with diagonal $h_{i j}$ and $g_{i j}=\delta_{i j}$ as mentioned above, $F^{i j}$ is diagonal. For $F=-K^{-1}$, we have $F^{i j}=K^{-1} \tilde{h}^{i j}=K^{-1} \lambda_{i}^{-1} g^{i j}$.

Recall, see e.g. $[14,17,20,22]$, that for a hypersurface moving according to $\frac{d}{d t} X=$ $-F \nu$, we have

$$
\begin{aligned}
\frac{d}{d t} g_{i j} & =-2 F h_{i j}, \\
\frac{d}{d t} h_{i j} & =F_{; i j}-F h_{i}^{k} h_{k j}, \\
\frac{d}{d t} \nu^{\alpha} & =g^{i j} F_{; i} X_{; j}^{\alpha},
\end{aligned}
$$

where Greek indices refer to components in the ambient space $\mathbb{R}^{3}$. In order to compute evolution equations, we will need the Gauß equation and the Ricci identity for the second fundamental form

$$
\begin{aligned}
R_{i j k l} & =h_{i k} h_{j l}-h_{i l} h_{j k}, \\
h_{i k ; l j} & =h_{i k ; j l}+h_{k}^{a} R_{a i l j}+h_{i}^{a} R_{a k l j} .
\end{aligned}
$$

We will also employ the Gauß formula and the Weingarten equation

$$
X_{; i j}^{\alpha}=-h_{i j} \nu^{\alpha} \quad \text { and } \quad \nu_{; i}^{\alpha}=h_{i}^{k} X_{; k}^{\alpha} .
$$

For tensors $A$ and $B, A_{i j} \geq B_{i j}$ means that $\left(A_{i j}-B_{i j}\right)$ is positive definite. Finally, we use $c$ to denote universal, estimated constants.

\section{A Monotone QuAntity}

Theorem 3.1. For a family of smooth closed strictly convex surfaces $M_{t}$ in $\mathbb{R}^{3}$ flowing according to $\dot{X}=\frac{1}{K} \nu$,

$$
\max _{M_{t}} \frac{\left(\lambda_{1}-\lambda_{2}\right)^{2}}{4 \lambda_{1}^{2} \lambda_{2}^{2}}=\max _{M_{t}} \frac{2|A|^{2}-H^{2}}{\left(H^{2}-|A|^{2}\right)^{2}} \equiv \max _{M_{t}} w
$$

is non-increasing in time.

An immediate consequence of this theorem is 
Corollary 3.2. The only homothetically expanding smooth closed strictly convex surfaces $M_{t}$, solving the flow equation $\dot{X}=\frac{1}{K} \nu$ in $\mathbb{R}^{3}$, are spheres.

Proof. The quantity $\frac{\left(\lambda_{1}-\lambda_{2}\right)^{2}}{\lambda_{1}^{2} \lambda_{2}^{2}}$ is negative homogeneous of degree two in the principal curvatures and non-negative. If $M$ is homothetically expanding, Theorem 3.1 implies that $\left(\lambda_{1}-\lambda_{2}\right)^{2}=0$ everywhere. Thus $M_{t}$ is umbilic and [27, Lemma 7.1] implies that $M_{t}$ is a sphere.

Proof of Theorem 3.1. First and second derivatives of $F=-1 / K$ with respect to the second fundamental form are given by

$$
\begin{aligned}
F^{i j} & =\frac{1}{K} \tilde{h}^{i j}, \\
F^{i j, k l} & =-\frac{1}{K}\left(\tilde{h}^{i j} \tilde{h}^{k l}+\tilde{h}^{i k} \tilde{h}^{j l}\right) .
\end{aligned}
$$

We combine (2.1), (2.2), (2.4), and (2.5) in order to get the following evolution equations

$$
\frac{d}{d t} H-F^{i j} H_{; i j}=\frac{1}{K}\left(H^{2}-3|A|^{2}\right)-\frac{1}{K} \sum \frac{1}{\lambda_{i} \lambda_{j}}\left(h_{i i ; k} h_{j j ; k}+h_{i j ; k}^{2}\right)
$$

and

$$
\begin{aligned}
\frac{d}{d t}|A|^{2}-F^{i j}\left(|A|^{2}\right)_{; i j}= & \frac{1}{K}\left(2 H|A|^{2}-6 \operatorname{tr} A^{3}\right)-\frac{1}{K} \sum \frac{2}{\lambda_{k}} h_{i j ; k}^{2} \\
& -\frac{1}{K} \sum \frac{2 \lambda_{k}}{\lambda_{i} \lambda_{j}}\left(h_{i i ; k} h_{j j ; k}+h_{i j ; k}^{2}\right)
\end{aligned}
$$

For the rest of the proof, we consider a critical point of $\left.w\right|_{M_{t}}$ for some $t>0$, where $w>0$ and $\lambda_{1} \neq \lambda_{2}$. It suffices to show that $w$ is non-increasing at such a point. Then our theorem follows.

We differentiate $w$

$$
\begin{aligned}
& w=\frac{-H^{2}+2|A|^{2}}{\left(H^{2}-|A|^{2}\right)^{2}}, \\
& w_{; i}=\frac{2 H\left(H^{2}-3|A|^{2}\right) H_{; i}+2|A|^{2}\left(|A|^{2}\right)_{; i}}{\left(H^{2}-|A|^{2}\right)^{3}}, \\
& w_{; i j}=\frac{1}{\left(H^{2}-|A|^{2}\right)^{4}}\left\{2 H\left(H^{2}-|A|^{2}\right)\left(H^{2}-3|A|^{2}\right) H_{; i j}\right. \\
& +2|A|^{2}\left(H^{2}-|A|^{2}\right)\left(|A|^{2}\right)_{; i j} \\
& \left(6\left(H^{2}-|A|^{2}\right)^{2}-12 H^{2}\left(H^{2}-3|A|^{2}\right)\right) H_{; i} H_{; j} \\
& +2\left(H^{2}+2|A|^{2}\right)\left(|A|^{2}\right)_{; i}\left(|A|^{2}\right)_{; j} \\
& \left.-12 H|A|^{2}\left(H_{; i}\left(|A|^{2}\right)_{; j}+H_{; j}\left(|A|^{2}\right)_{; i}\right)\right\} .
\end{aligned}
$$

In a critical point of $w$ with $\lambda_{1} \neq \lambda_{2}$, we get

$$
\begin{aligned}
0 & =4 \lambda_{2}^{2}\left(\lambda_{1}-\lambda_{2}\right) h_{11 ; k}+4 \lambda_{1}^{2}\left(\lambda_{2}-\lambda_{1}\right) h_{22 ; k} \quad \text { for } k=1,2, \\
h_{22 ; 1} & =\frac{\lambda_{2}^{2}}{\lambda_{1}^{2}} h_{11 ; 1} \equiv a_{1} h_{11 ; 1} \quad \text { and } \quad h_{11 ; 2}=\frac{\lambda_{1}^{2}}{\lambda_{2}^{2}} h_{22 ; 2} \equiv a_{2} h_{22 ; 2} .
\end{aligned}
$$


The evolution equation of $w$ is

$$
\begin{aligned}
\left(H^{2}-|A|^{2}\right)^{4} & \cdot\left(\frac{d}{d t} w-F^{i j} w_{; i j}\right)= \\
= & 2 H\left(H^{2}-|A|^{2}\right)\left(H^{2}-3|A|^{2}\right)\left(\frac{d}{d t} H-F^{i j} H_{; i j}\right) \\
& +2|A|^{2}\left(H^{2}-|A|^{2}\right)\left(\frac{d}{d t}|A|^{2}-F^{i j}\left(|A|^{2}\right)_{; i j}\right) \\
& +\left(6\left(H^{2}-|A|^{2}\right)^{2}-12 H^{2}\left(H^{2}-3|A|^{2}\right)\right)\left(-F^{i j} H_{; i} H_{; j}\right) \\
& +2\left(H^{2}+2|A|^{2}\right)\left(-F^{i j}\left(|A|^{2}\right)_{; i}\left(|A|^{2}\right)_{; j}\right) \\
& -12 H|A|^{2}\left(-F^{i j}\left(H_{; i}\left(|A|^{2}\right)_{; j}+H_{; j}\left(|A|^{2}\right)_{; i}\right)\right) .
\end{aligned}
$$

We consider the terms on the right-hand side individually and use (3.3), (3.4), and $(3.5)$

$$
\begin{aligned}
& \frac{d}{d t} H-F^{i j} H_{; i j}=-\frac{2\left(\lambda_{1}^{2}-\lambda_{1} \lambda_{2}+\lambda_{2}^{2}\right)}{\lambda_{1} \lambda_{2}} \\
& -\frac{2\left(\lambda_{1}^{2} a_{1}^{2}+\lambda_{1} \lambda_{2} a_{1}^{2}+\lambda_{1} \lambda_{2} a_{1}+\lambda_{2}^{2}\right)}{\lambda_{1}^{3} \lambda_{2}^{3}} h_{11 ; 1}^{2} \\
& -\frac{2\left(\lambda_{2}^{2} a_{2}^{2}+\lambda_{2} \lambda_{1} a_{2}^{2}+\lambda_{2} \lambda_{1} a_{2}+\lambda_{1}^{2}\right)}{\lambda_{2}^{3} \lambda_{1}^{3}} h_{22 ; 2}^{2} \\
& =-\frac{2\left(\lambda_{1}^{2}-\lambda_{1} \lambda_{2}+\lambda_{2}^{2}\right)}{\lambda_{1} \lambda_{2}} \\
& -\frac{2\left(\lambda_{1}+\lambda_{2}\right)\left(\lambda_{1}^{2}+\lambda_{2}^{2}\right)}{\lambda_{1} \lambda_{2}}\left(\frac{1}{\lambda_{1}^{5}} h_{11 ; 1}^{2}+\frac{1}{\lambda_{2}^{5}} h_{22 ; 2}^{2}\right), \\
& 2 H\left(H^{2}-|A|^{2}\right)\left(H^{2}-3|A|^{2}\right) \cdot\left(\frac{d}{d t} H-F^{i j} H_{; i j}\right)= \\
& =-8\left(\lambda_{1}+\lambda_{2}\right)\left(\lambda_{1}^{2}-\lambda_{1} \lambda_{2}+\lambda_{2}^{2}\right) \lambda_{1} \lambda_{2}\left(\frac{d}{d t} H-F^{i j} H_{; i j}\right) \\
& =16\left(\lambda_{1}+\lambda_{2}\right)\left(\lambda_{1}^{2}-\lambda_{1} \lambda_{2}+\lambda_{2}^{2}\right)^{2} \\
& +16\left(\lambda_{1}+\lambda_{2}\right)^{2}\left(\lambda_{1}^{2}-\lambda_{1} \lambda_{2}+\lambda_{2}^{2}\right)\left(\lambda_{1}^{2}+\lambda_{2}^{2}\right) . \\
& \cdot\left(\frac{1}{\lambda_{1}^{5}} h_{11 ; 1}^{2}+\frac{1}{\lambda_{2}^{5}} h_{22 ; 2}^{2}\right) \\
& =8\left(\lambda_{1}+\lambda_{2}\right)\left(2 \lambda_{1}^{4}-4 \lambda_{1}^{3} \lambda_{2}+6 \lambda_{1}^{2} \lambda_{2}^{2}-4 \lambda_{1} \lambda_{2}^{3}+2 \lambda_{2}^{4}\right) \\
& +\left(4 \lambda_{1}^{7} \lambda_{2}+4 \lambda_{1}^{6} \lambda_{2}^{2}+4 \lambda_{1}^{5} \lambda_{2}^{3}+8 \lambda_{1}^{4} \lambda_{2}^{4}\right. \\
& \left.+4 \lambda_{1}^{3} \lambda_{2}^{5}+4 \lambda_{1}^{2} \lambda_{2}^{6}+4 \lambda_{1} \lambda_{2}^{7}\right) \\
& \text {. } \frac{4}{\lambda_{1} \lambda_{2}}\left(\frac{1}{\lambda_{1}^{5}} h_{11 ; 1}^{2}+\frac{1}{\lambda_{2}^{5}} h_{22 ; 2}^{2}\right) \text {, } \\
& \frac{d}{d t}|A|^{2}-F^{i j}\left(|A|^{2}\right)_{; i j}=-\frac{2\left(\lambda_{1}+\lambda_{2}\right)\left(2 \lambda_{1}^{2}-3 \lambda_{1} \lambda_{2}+2 \lambda_{2}^{2}\right)}{\lambda_{1} \lambda_{2}}
\end{aligned}
$$




$$
\begin{aligned}
& \text { SURFACES EXPANDING BY THE INVERSE GAUSS CURVATURE FLOW } \\
& -\frac{2\left(2 \lambda_{1}^{2} a_{1}^{2}+2 \lambda_{1} \lambda_{2} a_{1}^{2}+3 \lambda_{2}^{2} a_{1}^{2}+2 \lambda_{1} \lambda_{2} a_{1}+3 \lambda_{2}^{2}\right)}{\lambda_{1}^{2} \lambda_{2}^{3}} h_{11 ; 1}^{2} \\
& -\frac{2\left(2 \lambda_{2}^{2} a_{2}^{2}+2 \lambda_{2} \lambda_{1} a_{2}^{2}+3 \lambda_{1}^{2} a_{2}^{2}+2 \lambda_{2} \lambda_{1} a_{2}+3 \lambda_{1}^{2}\right)}{\lambda_{2}^{2} \lambda_{1}^{3}} h_{22 ; 2}^{2} \\
& =-\frac{2\left(\lambda_{1}+\lambda_{2}\right)\left(2 \lambda_{1}^{2}-3 \lambda_{1} \lambda_{2}+2 \lambda_{2}^{2}\right)}{\lambda_{1} \lambda_{2}} \\
& -\frac{2\left(3 \lambda_{1}^{4}+2 \lambda_{1}^{3} \lambda_{2}+2 \lambda_{1}^{2} \lambda_{2}^{2}+2 \lambda_{1} \lambda_{2}^{3}+3 \lambda_{2}^{4}\right)}{\lambda_{1} \lambda_{2}} . \\
& \cdot\left(\frac{1}{\lambda_{1}^{5}} h_{11 ; 1}^{2}+\frac{1}{\lambda_{2}^{5}} h_{22 ; 2}^{2}\right) \\
& 2|A|^{2}\left(H^{2}-|A|^{2}\right) \cdot\left(\frac{d}{d t}|A|^{2}-F^{i j}\left(|A|^{2}\right)_{; i j}\right)= \\
& =4\left(\lambda_{1}^{2}+\lambda_{2}^{2}\right) \lambda_{1} \lambda_{2}\left(\frac{d}{d t}|A|^{2}-F^{i j}\left(|A|^{2}\right)_{; i j}\right) \\
& =-8\left(\lambda_{1}+\lambda_{2}\right)\left(\lambda_{1}^{2}+\lambda_{2}^{2}\right)\left(2 \lambda_{1}^{2}-3 \lambda_{1} \lambda_{2}+2 \lambda_{2}^{2}\right) \\
& -8\left(\lambda_{1}^{2}+\lambda_{2}^{2}\right)\left(3 \lambda_{1}^{4}+2 \lambda_{1}^{3} \lambda_{2}+2 \lambda_{1}^{2} \lambda_{2}^{2}+2 \lambda_{1} \lambda_{2}^{3}+3 \lambda_{2}^{4}\right) . \\
& \cdot\left(\frac{1}{\lambda_{1}^{5}} h_{11 ; 1}^{2}+\frac{1}{\lambda_{2}^{5}} h_{22 ; 2}^{2}\right) \\
& =-8\left(\lambda_{1}+\lambda_{2}\right)\left(2 \lambda_{1}^{4}-3 \lambda_{1}^{3} \lambda_{2}+4 \lambda_{1}^{2} \lambda_{2}^{2}-3 \lambda_{1} \lambda_{2}^{3}+2 \lambda_{2}^{4}\right) \\
& -\left(6 \lambda_{1}^{7} \lambda_{2}+4 \lambda_{1}^{6} \lambda_{2}^{2}+10 \lambda_{1}^{5} \lambda_{2}^{3}+8 \lambda_{1}^{4} \lambda_{2}^{4}+10 \lambda_{1}^{3} \lambda_{2}^{5}\right. \\
& \left.+4 \lambda_{1}^{2} \lambda_{2}^{6}+6 \lambda_{1} \lambda_{2}^{7}\right) \frac{4}{\lambda_{1} \lambda_{2}}\left(\frac{1}{\lambda_{1}^{5}} h_{11 ; 1}^{2}+\frac{1}{\lambda_{2}^{5}} h_{22 ; 2}^{2}\right) .
\end{aligned}
$$

For the remaining terms, we employ (3.2)

$$
\begin{aligned}
-F^{i j} H_{; i} H_{; j}= & -\frac{\left(a_{1}+1\right)^{2}}{\lambda_{1}^{2} \lambda_{2}} h_{11 ; 1}^{2}-\frac{\left(a_{2}+1\right)^{2}}{\lambda_{2}^{2} \lambda_{1}} h_{22 ; 2}^{2} \\
= & -\frac{\left(\lambda_{1}^{2}+\lambda_{2}^{2}\right)^{2}}{\lambda_{1} \lambda_{2}}\left(\frac{1}{\lambda_{1}^{5}} h_{11 ; 1}^{2}+\frac{1}{\lambda_{2}^{5}} h_{22 ; 2}^{2}\right) \\
\left(6\left(H^{2}-|A|^{2}\right)^{2}-12\right. & \left.H^{2}\left(H^{2}-3|A|^{2}\right)\right)\left(-F^{i j} H_{; i} H_{; j}\right)= \\
=- & \left(\lambda_{1}^{4}+\lambda_{1}^{3} \lambda_{2}+\lambda_{1}^{2} \lambda_{2}^{2}+\lambda_{1} \lambda_{2}^{3}+\lambda_{2}^{4}\right) \cdot \\
& \cdot 6\left(\lambda_{1}^{2}+\lambda_{2}^{2}\right)^{2} \frac{4}{\lambda_{1} \lambda_{2}}\left(\frac{1}{\lambda_{1}^{5}} h_{11 ; 1}^{2}+\frac{1}{\lambda_{2}^{5}} h_{22 ; 2}^{2}\right) \\
=- & \left(6 \lambda_{1}^{8}+6 \lambda_{1}^{7} \lambda_{2}+18 \lambda_{1}^{6} \lambda_{2}^{2}+18 \lambda_{1}^{5} \lambda_{2}^{3}+24 \lambda_{1}^{4} \lambda_{2}^{4}+18 \lambda_{1}^{3} \lambda_{2}^{5}\right. \\
& \left.+18 \lambda_{1}^{2} \lambda_{2}^{6}+6 \lambda_{1} \lambda_{2}^{7}+6 \lambda_{2}^{8}\right) \cdot \\
& \cdot \frac{4}{\lambda_{1} \lambda_{2}}\left(\frac{1}{\lambda_{1}^{5}} h_{11 ; 1}^{2}+\frac{1}{\lambda_{2}^{5}} h_{22 ; 2}^{2}\right) \\
= & \frac{4\left(\lambda_{2} a_{1}+\lambda_{1}\right)^{2}}{\lambda_{1}^{2} \lambda_{2}} h_{11 ; 1}^{2}-\frac{4\left(\lambda_{1} a_{2}+\lambda_{2}\right)^{2}}{\lambda_{2}^{2} \lambda_{1}} h_{22 ; 2}^{2} \\
-F^{i j}\left(|A|^{2}\right)_{; i}\left(|A|^{2}\right)_{; j}=- & \left(\lambda_{1}^{3}+\lambda_{2}^{3}\right)^{2} \cdot \frac{4}{\lambda_{1} \lambda_{2}}\left(\frac{1}{\lambda_{1}^{5}} h_{11 ; 1}^{2}+\frac{1}{\lambda_{2}^{5}} h_{22 ; 2}^{2}\right)
\end{aligned}
$$




$$
\begin{aligned}
& 2\left(H^{2}+2|A|^{2}\right) \cdot\left(-F^{i j}\left(|A|^{2}\right)_{; i}\left(|A|^{2}\right)_{; j}\right)= \\
& =-2\left(3 \lambda_{1}^{2}+2 \lambda_{1} \lambda_{2}+3 \lambda_{2}^{2}\right)\left(\lambda_{1}^{3}+\lambda_{2}^{3}\right)^{2} . \\
& \cdot \frac{4}{\lambda_{1} \lambda_{2}}\left(\frac{1}{\lambda_{1}^{5}} h_{11 ; 1}^{2}+\frac{1}{\lambda_{2}^{5}} h_{22 ; 2}^{2}\right) \\
& =-\left(6 \lambda_{1}^{8}+4 \lambda_{1}^{7} \lambda_{2}+6 \lambda_{1}^{6} \lambda_{2}^{2}+12 \lambda_{1}^{5} \lambda_{2}^{3}+8 \lambda_{1}^{4} \lambda_{2}^{4}+12 \lambda_{1}^{3} \lambda_{2}^{5}\right. \\
& \left.+6 \lambda_{1}^{2} \lambda_{2}^{6}+4 \lambda_{1} \lambda_{2}^{7}+6 \lambda_{2}^{8}\right) \frac{4}{\lambda_{1} \lambda_{2}}\left(\frac{1}{\lambda_{1}^{5}} h_{11 ; 1}^{2}+\frac{1}{\lambda_{2}^{5}} h_{22 ; 2}^{2}\right), \\
& -F^{i j}\left(H_{; i}\left(|A|^{2}\right)_{; j}+H_{; j}\left(|A|^{2}\right)_{; i}\right)= \\
& =-\frac{4\left(\lambda_{2} a_{1}+\lambda_{1}\right)\left(a_{1}+1\right)}{\lambda_{1}^{2} \lambda_{2}} h_{11 ; 1}^{2}-\frac{4\left(\lambda_{1} a_{2}+\lambda_{2}\right)\left(a_{2}+1\right)}{\lambda_{2}^{2} \lambda_{1}} h_{22 ; 2}^{2} \\
& =-\left(\lambda_{1}^{3}+\lambda_{2}^{3}\right)\left(\lambda_{1}^{2}+\lambda_{2}^{2}\right) \cdot \frac{4}{\lambda_{1} \lambda_{2}}\left(\frac{1}{\lambda_{1}^{5}} h_{11 ; 1}^{2}+\frac{1}{\lambda_{2}^{5}} h_{22 ; 2}^{2}\right), \\
& -12 H|A|^{2}\left(-F^{i j}\left(H_{; i}\left(|A|^{2}\right)_{; j}+H_{; j}\left(|A|^{2}\right)_{; i}\right)\right)= \\
& =12\left(\lambda_{1}+\lambda_{2}\right)\left(\lambda_{1}^{2}+\lambda_{2}^{2}\right)^{2}\left(\lambda_{1}^{3}+\lambda_{2}^{3}\right) \text {. } \\
& \cdot \frac{4}{\lambda_{1} \lambda_{2}}\left(\frac{1}{\lambda_{1}^{5}} h_{11 ; 1}^{2}+\frac{1}{\lambda_{2}^{5}} h_{22 ; 2}^{2}\right) \\
& =\left(12 \lambda_{1}^{8}+12 \lambda_{1}^{7} \lambda_{2}+24 \lambda_{1}^{6} \lambda_{2}^{2}+36 \lambda_{1}^{5} \lambda_{2}^{3}+24 \lambda_{1}^{4} \lambda_{2}^{4}+36 \lambda_{1}^{3} \lambda_{2}^{5}\right. \\
& \left.+24 \lambda_{1}^{2} \lambda_{2}^{6}+12 \lambda_{1} \lambda_{2}^{7}+12 \lambda_{2}^{8}\right) \cdot \\
& \cdot \frac{4}{\lambda_{1} \lambda_{2}}\left(\frac{1}{\lambda_{1}^{5}} h_{11 ; 1}^{2}+\frac{1}{\lambda_{2}^{5}} h_{22 ; 2}^{2}\right) \text {. }
\end{aligned}
$$

Combining these expressions yields

$$
\begin{aligned}
\left(H^{2}-|A|^{2}\right)^{4}\left(\frac{d}{d t}\right. & \left.w-F^{i j} w_{i j}\right)= \\
& =-8\left(\lambda_{1}+\lambda_{2}\right)\left(\lambda_{1}-\lambda_{2}\right)^{2} \lambda_{1} \lambda_{2}-\frac{32 \lambda_{2}^{3}}{\lambda_{1}^{2}} h_{11 ; 1}^{2}-\frac{32 \lambda_{1}^{3}}{\lambda_{2}^{2}} h_{22 ; 2}^{2}, \\
\frac{d}{d t} w-F^{i j} w_{; i j} & =-\frac{\left(\lambda_{1}+\lambda_{2}\right)\left(\lambda_{1}-\lambda_{2}\right)^{2}}{2 \lambda_{1}^{3} \lambda_{2}^{3}}-\frac{2}{\lambda_{1}^{6} \lambda_{2}} h_{11 ; 1}^{2}-\frac{2}{\lambda_{1} \lambda_{2}^{6}} h_{22 ; 2}^{2} \\
\leq & 0 .
\end{aligned}
$$

We finally apply the maximum principle and our theorem follows.

\section{Convergence to Infinity}

It is known [17], that (1.1) is a parabolic evolution equation for strictly convex initial data and that it has a solution on a maximal time interval $[0, T)$. Here, we want to show that some points on $M_{t}$ converge to infinity for $t \uparrow T$, i. e.

$$
\lim _{t \uparrow T} \sup _{M_{t}}|X|=\infty .
$$

We show that the principal curvatures of $M_{t}$ stay uniformly bounded above.

Lemma 4.1. For a smooth closed strictly convex surface $M$ in $\mathbb{R}^{3}$, flowing according to $\dot{X}=\frac{1}{K} \nu$, the maximum of the principal curvatures is non-increasing. 
Proof. Consider $M_{i j}=h_{i j}-\mu g_{i j}$ with $\mu>0$ so large that $M_{i j}$ is negative semidefinite for some time $t_{0}$. We wish to show that $M_{i j}$ is negative semi-definite for $t>t_{0}$. Combine (2.2), (2.4), and (2.5) to obtain

$$
\frac{d}{d t} h_{i j}-F^{k l} h_{i j ; k l}=\frac{1}{K} H h_{i j}-\frac{1}{K} h_{i}^{k} h_{k j}-\frac{1}{K}\left(\tilde{h}^{k l} \tilde{h}^{r s}+\tilde{h}^{k r} \tilde{h}^{l s}\right) h_{k l ; i} h_{r s ; j} .
$$

In the evolution equation for $M_{i j}$, we drop the negative definite terms involving derivatives of the second fundamental form

$$
\frac{d}{d t} M_{i j}-F^{k l} M_{i j ; k l} \leq \frac{1}{K}\left(H h_{i j}-h_{i}^{k} h_{k j}-2 \mu h_{i j}\right) .
$$

Let $\xi$ be a zero eigenvalue of $M_{i j}$ with $|\xi|=1, M_{i j} \xi^{j}=h_{i j} \xi^{j}-\mu g_{i j} \xi^{j}=0$. So we obtain in a point with $M_{i j} \leq 0$

$$
\left(H h_{i j}-h_{i}^{k} h_{k j}-2 \mu h_{i j}\right) \xi^{i} \xi^{j}=H \mu-3 \mu^{2} \leq 2 \mu \mu-3 \mu^{2} \leq 0
$$

and the maximum principle for tensors $[8,13]$ implies the claimed result.

We obtain a pinching estimate

Lemma 4.2. For a smooth closed strictly convex surface $M_{t}$ in $\mathbb{R}^{3}$, flowing according to $\dot{X}=\frac{1}{K} \nu$, there exists $c=c\left(M_{0}\right)$ such that $0<\frac{1}{c} \leq \frac{\lambda_{1}}{\lambda_{2}} \leq c$.

Proof. Choose $\mu>0$ such that $\lambda_{1}, \lambda_{2} \leq \mu$ at $t=0$. Theorem 3.1 and Lemma 4.1 imply that

$$
\frac{1}{\mu^{2}} \frac{\left(\frac{\lambda_{1}}{\lambda_{2}}-1\right)^{2}}{\frac{\lambda_{1}}{\lambda_{2}}}=\frac{1}{\mu^{2}} \frac{\left(\lambda_{1}-\lambda_{2}\right)^{2}}{\lambda_{1} \lambda_{2}} \leq \frac{\left(\lambda_{1}-\lambda_{2}\right)^{2}}{\lambda_{1}^{2} \lambda_{2}^{2}} \leq c .
$$

We obtain the bound on $\frac{\lambda_{1}}{\lambda_{2}}$ claimed above.

It is only here that we use the monotone quantity of Theorem 3.1. For our purposes, this quantity is better than scaling invariant. As it becomes apparent from the proof of Lemma 5.1, however, the Aleksandrov reflection principle can be used instead for the rest of the proof. This simplifies the proof compared to $[5,21]$, where similar monotone quantities are used. Later on, see Theorem 7.1 and Theorem 7.6, we will use monotone quantities to improve the convergence rate that follows from Aleksandrov reflection.

The next result shows that $K$ stays uniformly bounded below by a positive constant as long as $M_{t}$ is enclosed by a ball of fixed positive radius. For similar results see [28] and [10, Proposition 4.13].

Lemma 4.3. For a strictly convex solution of (1.1), $K$ is uniformly bounded below by a positive constant in terms of the radius $R$ of an enclosing sphere $B_{R}\left(x_{0}\right)$, the pinching ratio $\lambda_{1} / \lambda_{2}$, and $\max _{M_{0}} \frac{K^{-1}}{2 R-\left\langle X-x_{0}, \nu\right\rangle}$. More precisely, we have everywhere

$$
K \geq \frac{1}{3 R} \min \left\{\left(\max _{M_{0}} \frac{K^{-1}}{2 R-\left\langle X-x_{0}, \nu\right\rangle}\right)^{-1}, \frac{\varepsilon^{2}}{4 R}\right\},
$$

where $\varepsilon=\frac{1}{1+c}$ with $c$ as in Lemma 4.2. We obtain a positive lower bound on the principal curvatures. 
Proof. We may assume that $x_{0}=0$. Observe that $3 R \geq 2 R-\langle X, \nu\rangle \geq R$. Thus $\frac{K^{-1}}{2 R-\langle X, \nu\rangle}$ is finite for strictly convex surfaces.

Standard computations $[14,17,20,22]$ yield the evolution equations

$$
\begin{aligned}
\frac{d}{d t} X^{\beta}-F^{i j} X_{; i j}^{\beta} & =\frac{3}{K} \nu^{\beta}, \\
\frac{d}{d t} \nu^{\beta}-F^{i j} \nu_{; i j}^{\beta} & =\frac{H}{K} \nu^{\beta}, \\
\frac{d}{d t}\langle X, \nu\rangle-F^{i j}\langle X, \nu\rangle_{; i j} & =-\frac{1}{K}+\frac{H}{K}\langle X, \nu\rangle, \\
\frac{d}{d t} \frac{1}{K}-F^{i j}\left(\frac{1}{K}\right)_{; i j} & =\frac{H}{K^{2}} .
\end{aligned}
$$

In a critical point of $\left.\frac{K^{-1}}{2 R-\langle X, \nu\rangle}\right|_{M_{t}}$, we obtain

$$
\frac{d}{d t} \log \frac{K^{-1}}{2 R-\langle X, \nu\rangle}-F^{i j}\left(\log \frac{K^{-1}}{2 R-\langle X, \nu\rangle}\right)_{; i j}=\frac{1}{2 R-\langle X, \nu\rangle} \frac{1}{K}(2 R H-1) .
$$

So we get in an increasing maximum

$$
\begin{aligned}
0 & \leq 2 R H-1, \\
\frac{\varepsilon^{2}}{4 R^{2}} & \leq \varepsilon^{2} H^{2} \leq K
\end{aligned}
$$

and deduce there that

$$
\frac{K^{-1}}{2 R-\langle X, \nu\rangle} \leq \frac{4 \varepsilon^{-2} R^{2}}{R}
$$

Thus we obtain everywhere

$$
\frac{K^{-1}}{3 R} \leq \frac{K^{-1}}{2 R-\langle X, \nu\rangle} \leq \max \left\{\max _{M_{0}} \frac{K^{-1}}{2 R-\langle X, \nu\rangle}, \frac{4 R}{\varepsilon^{2}}\right\}
$$

and (4.1) follows.

Finally, the positive lower bound on $K$ and our pinching estimate, Lemma 4.2, imply a positive lower bound on the principal curvatures.

Let us recall a form of the maximum principle for evolving hypersurfaces.

Lemma 4.4. Let $M_{t}$ and $\tilde{M}_{t}$ be two smooth closed strictly convex solutions to (1.1) on some time interval $\left[0, T^{*}\right)$. If $M_{0}$ encloses $\tilde{M}_{0}$, then $M_{t}$ encloses $\tilde{M}_{t}$ at any time $t \in\left[0, T^{*}\right)$, for which both solutions exist.

Proof. This is a standard consequence of the maximum principle.

The next result describes the evolution of spheres.

Lemma 4.5. Spheres $\partial B_{r(t)}\left(x_{0}\right)$ solve (1.1) for $t \in[0, T)$ with $r(t)=(T-t)^{-1}$ and $T=r^{-1}(0)$.

Proof. The evolution equation for the radius of a sphere is

$$
\dot{r}(t)=r^{2}(t) \text {. }
$$

Lemma 4.6. Let $M_{t}$ be a family of smooth closed strictly convex solutions to (1.1) on a maximal time interval $[0, T)$. Then $T<\infty$. 
Proof. Spheres solving (1.1) tend to infinity in finite time, see Lemma 4.5. So Lemma 4.4 implies that $T<\infty$.

Lemma 4.7. Let $M_{t}$ be a family of smooth closed strictly convex surfaces solving (1.1) on a maximal time interval $[0, T)$. Then

$$
\lim _{t \uparrow T} \sup _{M_{t}}|X|=\infty \text {. }
$$

Proof. Assume that $M_{t} \subset B_{R}(0), 0 \leq t<T$, for some $R>0$. Then Lemmata 4.1 and 4.3 imply that the principal curvatures of $M_{t}$ stay uniformly bounded above and below by positive constants. Thus (1.1) can be rewritten as a uniformly parabolic equation and the estimates of Krylov, Safonov, Evans (see also [1]), and Schauder imply uniform a priori estimates up to $t=T$. This allows to continue the solution $M_{t}$ smoothly past $t=T$, a contradiction. Note finally, that (1.1) is an expanding flow, so $\sup _{M_{t}}|X|$ is monotone in $t$ and the limit exists.

\section{Convergence to a Sphere}

Lemma 5.1. Under the assumptions of Theorem 1.1, we get

$$
\liminf _{t \uparrow T}|X|=\infty,
$$

more precisely, there exists $c=c\left(M_{0}\right)$ such that

$$
\sup _{M_{t}}|X|-c \leq(T-t)^{-1} \leq \inf _{M_{t}}|X|+c
$$

and

$$
M_{t} \cdot(T-t) \subset B_{1+c(T-t)}(0) \backslash B_{1-c(T-t)}(0),
$$

so the rescaled surfaces $M_{t} \cdot(T-t)$ converge to the unit sphere $\mathbb{S}^{2}$ in Hausdorff distance.

Proof. We may shift the origin such that 0 lies inside $M_{0}$. This does not affect the convergence rate claimed above.

Define the support function $u: \mathbb{S}^{2} \times[0, T) \rightarrow \mathbb{R}_{+}$for a convex surface by

$$
u(z, t)=\left\langle X\left(\nu^{-1}(z), t\right), z\right\rangle .
$$

It fulfills the evolution equation, see e.g. [4],

$$
\dot{u}=\frac{\operatorname{det}\left(u_{; i j}+u \sigma_{i j}\right)}{\operatorname{det}\left(\sigma_{i j}\right)},
$$

where $u_{; i j}$ denotes covariant derivatives on $\mathbb{S}^{2}$ and $\sigma_{i j}$ is the standard metric on $\mathbb{S}^{2}$.

We apply the Aleksandrov reflection principle of Bennett Chow, Robert Gulliver [7], and James McCoy [19, Theorem 3.1] and obtain a uniform bound on the oscillation (and the gradient) of $u(\cdot, t)$ for all $t \in[0, T)$, that depends only on the initial data.

As $\sup _{M_{t}}|X| \rightarrow \infty$ for $t \uparrow T$, we obtain that $\inf _{M_{t}}|X| \rightarrow \infty$ for $t \uparrow T$, more precisely, we have

$$
\sup _{M_{t}}|X| \leq \inf _{M_{t}}|X|+c .
$$

It remains to show that

$$
\inf _{M_{t}}|X| \leq(T-t)^{-1} \leq \sup _{M_{t}}|X| .
$$


Consider the surfaces $\partial M_{(T-t)^{-1}}(0)$, solving (1.1). For $t=T, M_{t}$ and $\partial B_{(T-t)^{-1}}(0)$ converge to infinity. We claim that for all $t \in[0, T), \partial B_{(T-t)^{-1}}(0) \cap M_{t} \neq \emptyset$. Otherwise, for some $t_{0}, M_{t_{0}}$ encloses $\partial B_{(T-t)^{-1}}(0)$ or is contained in $B_{(T-t)^{-1}}(0)$. Both cases are similar. We only consider the first case. Choose $\varepsilon>0$ such that $M_{t_{0}}$ encloses also $\partial B_{\left(T-t_{0}-\varepsilon\right)^{-1}}(0)$, a slightly larger sphere. For $t \in\left[t_{0}, T-\varepsilon\right)$, $\partial B_{(T-t-\varepsilon)^{-1}}(0)$ solves (1.1). As $\partial B_{(T-t-\varepsilon)^{-1}}(0)$ converges to infinity for $t \uparrow T-\varepsilon$, Lemma 4.4 implies that $M_{t}$ has to converge to infinity for $t \uparrow T-\varepsilon$, a contradiction.

In terms of the support function $u$, this Lemma implies that

$$
u(x, t)-c \leq(T-t)^{-1} \leq u(x, t)+c .
$$

Note that this estimate is sharp for spheres $\partial B_{(T-t)^{-1}}(Q)$ solving (1.1), if $Q$ is different from the origin. The method of [5], see also [21], where the origin is replaced by some $q(t)$, can be adapted to the present situation. In order to improve the estimate (5.2), however, we need a monotone quantity similar to (1.2) with a better scaling behavior. We address this issue in Section 7 .

\section{Smooth Convergence to a Sphere}

Lemma 6.1. Under the assumptions of Theorem 1.1, there exists $c=c\left(M_{0}\right)$, such that

$$
K \leq c \cdot(T-t)^{2} .
$$

Proof. For $\mu \gg 1$ to be fixed below, we consider

$$
w:=|X|^{2}-\mu \frac{1}{K} .
$$

We may assume that $\mu$ is so large that $w<0$ on $M_{0}$. Our aim is to show that $w$ stays negative during the flow. We use the evolution equations of the proof of Lemma 4.3. The evolution equation of $w$ is given by

$$
\frac{d}{d t} w-F^{i j} w_{; i j}=6 \frac{1}{K}\langle X, \nu\rangle-2 \frac{H}{K^{2}}-\mu \frac{H}{K^{2}} .
$$

Let $t_{0} \in[0, T)$ be minimal such that $\max _{M_{t}} w=0$. Choose $x_{0} \in M_{t}$ such that $w\left(x_{0}, t_{0}\right)=0$. At this point, we apply the parabolic maximum principle and obtain

$$
\begin{aligned}
0 & \leq 6 K\langle X, \nu\rangle-(2+\mu) H & & \\
& \leq 6 K|X|-(2+\mu) H & & \\
& =6 \sqrt{K} \sqrt{\mu}-(2+\mu) H & & \text { as } w\left(x_{0}, t_{0}\right)=0, \\
& \leq(6 \sqrt{\mu}-2-\mu) H & & \text { as } K \leq H^{2} .
\end{aligned}
$$

We fix $\mu$ sufficiently large and obtain that $w \leq 0$ during the flow. In view of Lemma 5.1 , this implies the upper bound on the Gauß curvature claimed above.

Combining this result with the Lemmata 4.2, 4.3, and 5.1, we obtain

$$
\frac{1}{c} \cdot(T-t)^{2} \leq K \leq c \cdot(T-t)^{2} .
$$

We rescale our surfaces similarly as in [3]. Consider the embeddings $\tilde{X}(\cdot, t)$,

$$
\tilde{X}(z, t):=(T-t) \cdot X(z, t) .
$$


Define a new time function

$$
\tau(t):=-\log \left(\frac{T-t}{T}\right) .
$$

We use a tilde to denote geometric quantities of the rescaled surfaces. For $\tilde{X}$, we obtain the evolution equation

$$
\frac{d}{d \tau} \tilde{X}=\frac{1}{\tilde{K}} \tilde{\nu}-\tilde{X}
$$

Our a priori estimates and the estimates of Krylov, Safonov, Evans, and Schauder imply uniform bounds on all derivatives of the support function $\tilde{u}$ of $\tilde{X}$. Applying interpolation inequalities as in [23, Lemma C.2] to

$$
\begin{aligned}
|\tilde{u}-1| & \leq c \cdot(T-t), \\
\left|D^{k} \tilde{u}\right| & \leq c_{k},
\end{aligned}
$$

we get

$$
\left|D^{k} \tilde{u}\right| \leq c(k, \varepsilon) \cdot(T-t)^{1-\varepsilon}
$$

for any $\varepsilon>0$.

This finishes the proof of Theorem 1.1.

\section{Improved Convergence Rate}

Theorem 7.1. For a family of smooth closed strictly convex surfaces $M_{t}$ in $\mathbb{R}^{3}$ flowing according to $\dot{X}=\frac{1}{K} \nu$,

$$
\max _{M_{t}} \frac{\left(\lambda_{1}^{2}+\lambda_{2}^{2}\right)\left(\lambda_{1}-\lambda_{2}\right)^{2}}{8\left(\lambda_{1}+\lambda_{2}\right) \lambda_{1}^{3} \lambda_{2}^{3}} \equiv \max _{M_{t}} w \equiv \max _{M_{t}} \frac{|A|^{2}\left(2|A|^{2}-H^{2}\right)}{H\left(H^{2}-|A|^{2}\right)^{3}}
$$

is non-increasing in time.

Proof. We use Section 9 and obtain in a critical point of $w$

$$
\begin{aligned}
\frac{d}{d t} w-F^{i j} w_{; i j}= & \frac{-3\left(\lambda_{1}^{4}+2 \lambda_{1}^{3} \lambda_{2}-2 \lambda_{1}^{2} \lambda_{2}^{2}+2 \lambda_{1} \lambda_{2}^{3}+\lambda_{2}^{4}\right)\left(\lambda_{1}-\lambda_{2}\right)^{2}}{8\left(\lambda_{1}+\lambda_{2}\right)^{2} \lambda_{1}^{4} \lambda_{2}^{4}} \\
& -\frac{1}{\left(3 \lambda_{1}^{3}+3 \lambda_{1}^{2} \lambda_{2}-\lambda_{1} \lambda_{2}^{2}+3 \lambda_{2}^{3}\right)^{2}\left(\lambda_{1}+\lambda_{2}\right)^{2} \lambda_{1}^{7} \lambda_{2}^{2}} . \\
& \cdot\left(18 \lambda_{1}^{9}-9 \lambda_{1}^{8} \lambda_{2}+12 \lambda_{1}^{7} \lambda_{2}^{2}+72 \lambda_{1}^{6} \lambda_{2}^{3}-12 \lambda_{1}^{5} \lambda_{2}^{4}+70 \lambda_{1}^{4} \lambda_{2}^{5}\right. \\
& \left.\quad+60 \lambda_{1}^{3} \lambda_{2}^{6}+18 \lambda_{1} \lambda_{2}^{8}+27 \lambda_{2}^{9}\right) h_{11 ; 1}^{2} \\
- & \frac{1}{\left(3 \lambda_{2}^{3}+3 \lambda_{2}^{2} \lambda_{1}-\lambda_{2} \lambda_{1}^{2}+3 \lambda_{1}^{3}\right)^{2}\left(\lambda_{2}+\lambda_{1}\right)^{2} \lambda_{2}^{7} \lambda_{1}^{2}} . \\
& \cdot\left(18 \lambda_{2}^{9}-9 \lambda_{2}^{8} \lambda_{1}+12 \lambda_{2}^{7} \lambda_{1}^{2}+72 \lambda_{2}^{6} \lambda_{1}^{3}-12 \lambda_{2}^{5} \lambda_{1}^{4}+70 \lambda_{2}^{4} \lambda_{1}^{5}\right. \\
\leq 0 & \left.\quad+60 \lambda_{2}^{3} \lambda_{1}^{6}+18 \lambda_{2} \lambda_{1}^{8}+27 \lambda_{1}^{9}\right) h_{22 ; 2}^{2}
\end{aligned}
$$

We finally apply the maximum principle.

This allows to improve our bound on $\left|\lambda_{1}-\lambda_{2}\right|$. 
Lemma 7.2. For a smooth closed strictly convex surface $M_{t}$ in $\mathbb{R}^{3}$, flowing according to $\dot{X}=\frac{1}{K} \nu$, there exists a constant $c=c\left(M_{0}\right)$ such that $\left|\lambda_{1}-\lambda_{2}\right| \leq c \cdot K^{5 / 4} \leq$ $c \cdot(T-t)^{5 / 2}$.

Proof. This is a direct consequence of Theorem 7.1, Lemma 4.2 and Lemma 6.1.

We now closely follow the corresponding parts of [5] and [21].

Proposition 7.3. Define $q(t):=\frac{1}{4 \pi} \int_{M_{t}} K X$. Then

$$
\left|\langle X-q, \nu\rangle-\frac{1}{8 \pi} \int_{M_{t}} H\right| \leq \frac{1}{4 \pi} \cdot \sup _{M_{t}}\left|\lambda_{1}-\lambda_{2}\right| \cdot \mathcal{H}^{2}\left(M_{t}\right),
$$

where $\mathcal{H}^{2}\left(M_{t}\right)$ denotes the area of $M_{t}$.

Proof. This is [5, Proposition 4].

We will call $q(t)$ the pseudocenter of $M_{t}$.

We define $r_{+}(t)$ to be the minimal radius of a sphere, centered at $q(t)$, that encloses $M_{t}$. Similarly, we define $r_{-}(t)$ to be the maximal radius of a sphere, centered at $q(t)$, that is enclosed by $M_{t}$. Let $\rho_{-}(t)$ be the maximal radius of a sphere (with arbitrary center) enclosed by $M_{t}$ and $\rho_{+}(t)$ be the minimal radius of spheres enclosing $M_{t}$.

Lemma 7.4. Under the assumptions of Theorem 1.1, for $T-t$ sufficiently small, $r_{+}$and $r_{-}$are estimated as follows

$$
(T-t)^{-1} \cdot\left(1-c \cdot(T-t)^{3 / 2}\right) \leq r_{-}(t) \leq r_{+}(t) \leq(T-t)^{-1} \cdot\left(1+c \cdot(T-t)^{3 / 2}\right) .
$$

Proof. Denote the bounded component of $\mathbb{R}^{3} \backslash M_{t}$ by $E_{t}$. The transformation formula for integrals implies that

$$
\frac{1}{4 \pi} \int_{M_{t}} K X=\frac{1}{4 \pi} \int_{\mathbb{S}^{2}} X\left(\nu^{-1}(\cdot)\right) .
$$

So we see that $q(t) \in E_{t}$. We have

$$
\begin{aligned}
r_{+} & =\max _{M_{t}}\langle X-q(t), \nu\rangle, & & r_{-}=\min _{M_{t}}\langle X-q(t), \nu\rangle, \\
\rho_{+} & =\min _{p \in \mathbb{R}^{3}} \max _{M_{t}}\langle X-p, \nu\rangle, \quad \text { and } & \rho_{-} & =\max _{p \in E_{t}} \min _{M_{t}}\langle X-p, \nu\rangle .
\end{aligned}
$$

Recall the first variation formula for a vector field $Y$ along $M_{t}$

$$
\int_{M_{t}} H\langle Y, \nu\rangle=\int_{M_{t}} \operatorname{div}_{M_{t}} Y
$$

and get for $p \in E_{t}$ such that $\rho_{+}=\max _{M_{t}}\langle X-p, \nu\rangle$

$$
\int_{M_{t}} H \geq \frac{1}{\rho_{+}} \int_{M_{t}} H \cdot\langle X-p, \nu\rangle=\frac{1}{\rho_{+}} \int_{M_{t}} \operatorname{div}_{M_{t}} X=\frac{1}{\rho_{+}} \int_{M_{t}} 2=\frac{2}{\rho_{+}} \mathcal{H}^{2}\left(M_{t}\right) .
$$


We employ Proposition 7.3 and deduce that

$$
\begin{aligned}
r_{-} & \geq \frac{1}{8 \pi} \int_{M_{t}} H \cdot\left\{1-2\left(\int_{M_{t}} H\right)^{-1} \cdot \sup _{M_{t}}\left|\lambda_{1}-\lambda_{2}\right| \cdot \mathcal{H}^{2}\left(M_{t}\right)\right\} \\
& \geq \frac{1}{8 \pi} \int_{M_{t}} H \cdot\left\{1-\rho_{+} \cdot \sup _{M_{t}}\left|\lambda_{1}-\lambda_{2}\right|\right\} \\
& \geq \frac{1}{8 \pi} \int_{M_{t}} H \cdot\left\{1-c \cdot(T-t)^{3 / 2}\right\},
\end{aligned}
$$

where we have used the Lemmata 7.2 and 5.1. So we obtain

$$
r_{-}(t) \geq \frac{1}{8 \pi} \int_{M_{t}} H \cdot\left(1-c \cdot(T-t)^{3 / 2}\right)
$$

and similarly

$$
r_{+}(t) \leq \frac{1}{8 \pi} \int_{M_{t}} H \cdot\left(1+c \cdot(T-t)^{3 / 2}\right) .
$$

As in the proof of Lemma 5.1, we get

$$
r_{-} \leq \rho_{-} \leq(T-t)^{-1} \leq \rho_{+} \leq r_{+}
$$

and

$$
(T-t)^{-1} \cdot\left(1-c \cdot(T-t)^{3 / 2}\right) \leq \frac{1}{8 \pi} \int_{M_{t}} H \leq(T-t)^{-1} \cdot\left(1+c \cdot(T-t)^{3 / 2}\right) .
$$

Using (7.2) and (7.3) gives the claimed estimates on $r_{-}$and $r_{+}$.

Lemma 7.5. Under the assumptions of Theorem 1.1, $q(t)$ as defined in Proposition 7.3 is a smooth function of $t$ in $[0, T)$ and converges to some point $Q \in \mathbb{R}^{3}$ for $t \uparrow T$,

$$
|q(t)-Q| \leq c \cdot(T-t)^{1 / 2} .
$$

Proof. The definition of $q(t)$ involves only quantities that depend smoothly on $t$, so it remains to prove convergence for $t \uparrow T$.

For $0<t_{1}<t_{2}<T$, we want to estimate $\left|q\left(t_{1}\right)-q\left(t_{2}\right)\right|$ from above. We may assume that $q\left(t_{1}\right) \neq q\left(t_{2}\right)$. Consider the line passing through $q\left(t_{1}\right)$ and $q\left(t_{2}\right)$. It intersects the surface $M_{t_{2}}$ in two points, denoted by $p_{l}\left(t_{2}\right)$ and $p_{r}\left(t_{2}\right)$.

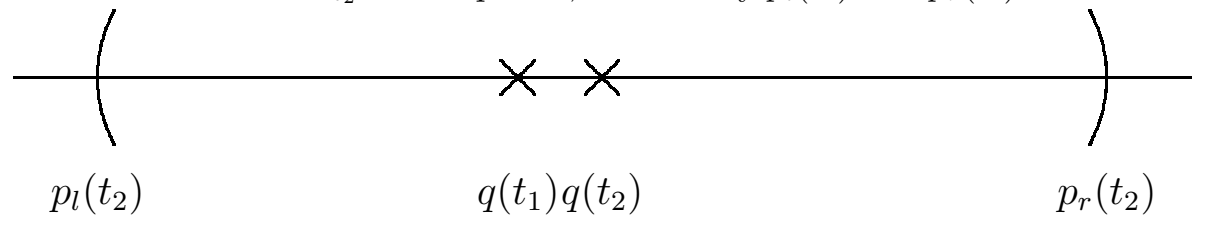

Figure: Convergence of pseudocenters

We may assume that

$$
\left\langle p_{r}\left(t_{2}\right)-q\left(t_{2}\right), q\left(t_{2}\right)-q\left(t_{1}\right)\right\rangle>0 .
$$


This corresponds to $p_{r}\left(t_{2}\right)$ and $q\left(t_{2}\right)$ lying on the same side of $q\left(t_{1}\right)$ as shown in the figure. We estimate

$$
\begin{array}{rlr}
2 c\left(T-t_{1}\right)^{1 / 2} \geq & r_{+}\left(t_{1}\right)-r_{-}\left(t_{1}\right) & \\
= & \operatorname{osc} u_{q\left(t_{1}\right)}\left(\cdot, t_{1}\right) & \text { by Lemma } 7.4 \\
\geq & \operatorname{osc} u_{q\left(t_{1}\right)}\left(\cdot, t_{2}\right) & \\
\geq & \left|p_{r}\left(t_{2}\right)-q\left(t_{1}\right)\right|-\left|p_{l}\left(t_{2}\right)-q\left(t_{1}\right)\right| & \\
= & \left|p_{r}\left(t_{2}\right)-q\left(t_{2}\right)\right|+\left|q\left(t_{2}\right)-q\left(t_{1}\right)\right| & \\
& -\left(\left|p_{l}\left(t_{2}\right)-q\left(t_{2}\right)\right|-\left|q\left(t_{2}\right)-q\left(t_{1}\right)\right|\right) & \\
\geq & 2\left|q\left(t_{2}\right)-q\left(t_{1}\right)\right|+r_{-}\left(t_{2}\right)-r_{+}\left(t_{2}\right) & \\
\geq & 2\left|q\left(t_{2}\right)-q\left(t_{1}\right)\right|-2 c\left(T-t_{2}\right)^{1 / 2}, &
\end{array}
$$

where $u_{q\left(t_{1}\right)}\left(\cdot, t_{2}\right)$ is the support function of $M_{t_{2}}-q\left(t_{1}\right)$. Thus we can apply Cauchy's convergence criterion. Finally, we let $t_{2} \uparrow T$ and the claimed bound follows.

This allows to improve our convergence result.

Theorem 7.6. Under the assumptions of Theorem 1.1, there exists $Q \in \mathbb{R}^{3}$ such that the Hausdorff distance of $M_{t}$ to a family of expanding spheres around $Q$ is bounded as follows

$$
d_{\mathcal{H}}\left(M_{t}, \partial B_{(T-t)^{-1}}(Q)\right) \leq c \cdot(T-t)^{1 / 2} .
$$

Proof. Combine the Lemmata 7.5 and 7.4.

Remark 7.7. Theorem 7.6 implies also better estimates for rescaled surfaces,

$$
d_{\mathcal{H}}\left(\left(M_{t}-Q\right) \cdot(T-t), \mathbb{S}^{2}\right) \leq c \cdot(T-t)^{3 / 2} .
$$

As above, this implies that all derivatives of the support function decay,

$$
\left\|u_{Q}(\cdot, t)-1\right\|_{C^{k}} \leq c(k, \varepsilon) \cdot(T-t)^{3 / 2-\varepsilon}
$$

for any $\varepsilon>0$, where $u_{Q}(\cdot, t)$ is the support function of $M_{t}-Q$. This implies for the principal curvatures $\tilde{\lambda}_{i}, i=1,2$, of $\left(M_{t}-Q\right) \cdot(T-t)$

$$
1-c(\varepsilon) \cdot(T-t)^{3 / 2-\varepsilon} \leq \tilde{\lambda}_{i} \leq 1+c(\varepsilon) \cdot(T-t)^{3 / 2-\varepsilon} .
$$

Without the additional $Q$, we get for expanding spheres $\partial B_{(T-t)^{-1}}(P)$,

$$
d_{\mathcal{H}}\left(\partial B_{(T-t)^{-1}}(P) \cdot(T-t), \mathbb{S}^{2}\right)=|P| \cdot(T-t)^{-1},
$$

so the estimate in Lemma 5.1 is sharp for $P \neq 0$.

In the proof of Theorem 1.1, we have used (1.2) only to prove that surfaces stay uniformly pinched, i.e. that $\lambda_{1} / \lambda_{2}$ is uniformly bounded. If we use it to bound $\left|\lambda_{1}-\lambda_{2}\right|$, and then $r_{+}$and $r_{-}$as above, we don't get more than in 5.1, where we used the oscillation estimates of [19]. Our computer program, however, did not yield a scaling invariant quantity that implies uniform pinching. This is similar to [2]. It might be possible to find a monotone quantity that allows to further improve this convergence rate. 


\section{Finding Monotone Quantities}

8.1. The Algorithm. We use a sieve algorithm and start with symmetric rational functions of the principal curvatures as candidates for test functions, e.g.

$$
w=\frac{p_{1}\left(\lambda_{1}, \lambda_{2}\right)}{p_{2}\left(\lambda_{1}, \lambda_{2}\right)}=\frac{\left(\lambda_{1}-\lambda_{2}\right)^{2}}{\lambda_{1}^{2} \lambda_{2}^{2}}
$$

Here, $p_{1} \neq 0$ and $p_{2} \neq 0$ are homogeneous polynomials.

In the end, we want to find functions $w$ such that $W:=\sup _{M_{t}} w$ is monotone and ensures convergence to round spheres.

We check, whether these test functions $w$ fulfill the following conditions.

(1) (a) $p_{1}\left(\lambda_{1}, \lambda_{2}\right), p_{2}\left(\lambda_{1}, \lambda_{2}\right) \geq 0$ for $0<\lambda_{1}, \lambda_{2}$,

(b) $p_{1}\left(\lambda_{1}, \lambda_{2}\right)=0$ for $\lambda_{1}=\lambda_{2}>0$.

(2) $\operatorname{deg} p_{1}<\operatorname{deg} p_{2}$.

(3) $\frac{\partial w\left(1, \lambda_{2}\right)}{\partial \lambda_{2}}<0$ for $0<\lambda_{2}<1$ and $\frac{\partial w\left(1, \lambda_{2}\right)}{\partial \lambda_{2}}>0$ for $\lambda_{2}>1$.

(4) $\frac{d}{d t} w-F^{i j} w_{; i j} \leq 0$

(a) for terms without derivatives of $\left(h_{i j}\right)$,

(b) for terms involving derivatives of $\left(h_{i j}\right)$, if $w_{; i}=0$ for $i=1,2$.

8.2. Motivation and Randomized Tests. For all flow equations considered, spheres contract to points and stay spherical. So we can only find monotone quantities, if $\operatorname{deg} p_{1} \geq \operatorname{deg} p_{2}$ or $p_{1}(\lambda, \lambda)=0$.

If $\operatorname{deg} p_{1} \geq \operatorname{deg} p_{2}$, we obtain that $W$ is non-increasing on any self-similarly expanding surface. So this does not imply convergence to a sphere.

Condition (3) ensures that the quantity decreases, if the eigenvalues approach each other.

In step (4a) and (4b), we check that we can apply the maximum principle. Here, we have to use various differentiation rules.

In steps (1), (2), and (3), inequalities are tested by evaluating both sides at random numbers. After enough testing, all candidates for which the above inequalities, evaluated at random numbers, were not violated, could be used to prove convergence to round spheres at infinity.

Alternatively, for surfaces, we can avoid using random numbers, compute evolution equations algebraically, and use Sturm's algorithm to test for non-negativity.

We expect that similar algorithms will be used to find monotone test functions for other (geometric) problems.

\section{Computing Evolution Equations}

It is straightforward to use a computer algebra system to obtain evolution equations of test quantities $w$, evaluated at a critical point of $w$. More precisely, let $M_{t}$ be a family of surfaces in $\mathbb{R}^{3}$, moving with normal velocity $F=F\left(\lambda_{1}, \lambda_{2}\right)$, where $F>0$ for contracting surfaces. Assume that the test quantity $w$ is a function of $H$ and $|A|^{2}$. Then $w$ fulfills the evolution equation

$$
\frac{d}{d t} w-F^{i j}(w)_{; i j}=C_{w}\left(\lambda_{1}, \lambda_{2}\right)+G_{w}\left(\lambda_{1}, \lambda_{2}\right) h_{11 ; 1}^{2}+G_{w}\left(\lambda_{2}, \lambda_{1}\right) h_{22 ; 2}^{2}
$$

in a critical point of $w$. It remains to compute $C_{w}$ ("constant terms") and $G_{w}$ ("gradient terms"). The following calculations are all similar as before and use 
(2.1), (2.2), (2.4), (2.5), and, see [3,12],

$$
F^{i j, k l} \eta_{i j} \eta_{k l}=\sum \frac{\partial^{2} F}{\partial \lambda_{i} \partial \lambda_{j}} \eta_{i i} \eta_{j j}+\sum_{i \neq j} \frac{\frac{\partial F}{\partial \lambda_{i}}-\frac{\partial F}{\partial \lambda_{j}}}{\lambda_{i}-\lambda_{j}}\left(\eta_{i j}\right)^{2},
$$

for symmetric matrices $\left(\eta_{i j}\right)$ and $\lambda_{1} \neq \lambda_{2}$ or $\lambda_{1}=\lambda_{2}$ and the last term is interpreted as a limit. For $w=H$, we obtain

$$
\begin{aligned}
C_{H} & =\left(\frac{\partial F}{\partial \lambda_{1}} \lambda_{1}^{2}+\frac{\partial F}{\partial \lambda_{2}} \lambda_{2}^{2}\right) H+\left(F-\frac{\partial F}{\partial \lambda_{1}} \lambda_{1}-\frac{\partial F}{\partial \lambda_{2}} \lambda_{2}\right)|A|^{2}, \\
G_{H} & =\frac{\partial^{2} F}{\partial \lambda_{1} \partial \lambda_{1}}+2 \frac{\partial^{2} F}{\partial \lambda_{1} \partial \lambda_{2}} a_{1}+\frac{\partial^{2} F}{\partial \lambda_{2} \partial \lambda_{2}} a_{1}^{2}+2 \frac{\frac{\partial F}{\partial \lambda_{1}}-\frac{\partial F}{\partial \lambda_{2}}}{\lambda_{1}-\lambda_{2}} a_{1}^{2},
\end{aligned}
$$

where

$$
a_{1}=-\frac{\partial w\left(\lambda_{1}+\lambda_{2}, \lambda_{1}^{2}+\lambda_{2}^{2}\right)}{\partial \lambda_{1}}\left(\frac{\partial w\left(\lambda_{1}+\lambda_{2}, \lambda_{1}^{2}+\lambda_{2}^{2}\right)}{\partial \lambda_{2}}\right)^{-1}
$$

is such that $h_{22 ; 1}=a_{1} h_{11 ; 1}$ in a critical point of $w$. Similarly, we get for $w=|A|^{2}$

$$
\begin{aligned}
C_{|A|^{2}}= & 2\left(\frac{\partial F}{\partial \lambda_{1}} \lambda_{1}^{2}+\frac{\partial F}{\partial \lambda_{2}} \lambda_{2}^{2}\right)|A|^{2}+2\left(F-\frac{\partial F}{\partial \lambda_{1}} \lambda_{1}-\frac{\partial F}{\partial \lambda_{2}} \lambda_{2}\right) \operatorname{tr} A^{3}, \\
G_{|A|^{2}}= & -2\left(\frac{\partial F}{\partial \lambda_{1}}\left(1+a_{1}^{2}\right)+2 \frac{\partial F}{\partial \lambda_{2}} a_{1}^{2}\right) \\
& +2\left(\frac{\partial^{2} F}{\partial \lambda_{1} \partial \lambda_{1}}+2 \frac{\partial^{2} F}{\partial \lambda_{1} \partial \lambda_{2}} a_{1}+\frac{\partial^{2} F}{\partial \lambda_{2} \partial \lambda_{2}} a_{1}^{2}\right) \lambda_{1}+4 \frac{\frac{\partial F}{\partial \lambda_{1}}-\frac{\partial F}{\partial \lambda_{2}}}{\lambda_{1}-\lambda_{2}} a_{1}^{2} \lambda_{2} .
\end{aligned}
$$

We also need some mixed terms

$$
\begin{aligned}
-F^{i j} H_{; i} H_{; j} & =-\frac{\partial F}{\partial \lambda_{1}}\left(1+a_{1}\right)^{2} h_{11 ; 1}^{2}-\frac{\partial F}{\partial \lambda_{2}}\left(1+a_{2}\right)^{2} h_{22 ; 2}^{2} \\
-F^{i j}\left(|A|^{2}\right)_{; i}\left(|A|^{2}\right)_{; j}= & -4 \frac{\partial F}{\partial \lambda_{1}}\left(\lambda_{1}+a_{1} \lambda_{2}\right)^{2} h_{11 ; 1}^{2}-4 \frac{\partial F}{\partial \lambda_{2}}\left(\lambda_{2}+a_{2} \lambda_{1}\right)^{2} h_{22 ; 2}^{2} \\
-2 F^{i j} H_{; i}\left(|A|^{2}\right)_{; j}= & -F^{i j}\left(H_{; i}\left(|A|^{2}\right)_{; j}+H_{; j}\left(|A|^{2}\right)_{; i}\right) \\
= & -4 \frac{\partial F}{\partial \lambda_{1}}\left(1+a_{1}\right)\left(\lambda_{1}+a_{1} \lambda_{2}\right) h_{11 ; 1}^{2} \\
& -4 \frac{\partial F}{\partial \lambda_{2}}\left(1+a_{2}\right)\left(\lambda_{2}+a_{2} \lambda_{1}\right) h_{22 ; 2}^{2} .
\end{aligned}
$$

Combining these expressions yields

$$
\begin{aligned}
C_{w}= & \frac{\partial w}{\partial H} C_{H}+\frac{\partial w}{\partial|A|^{2}} C_{|A|^{2}}, \\
G_{w}= & \frac{\partial w}{\partial H} G_{H}+\frac{\partial w}{\partial|A|^{2}} G_{|A|^{2}}-\frac{\partial^{2} w}{\partial H \partial H} \frac{\partial F}{\partial \lambda_{1}}\left(1+a_{1}\right)^{2} \\
& -4 \frac{\partial^{2} w}{\partial|A|^{2} \partial|A|^{2}} \frac{\partial F}{\partial \lambda_{1}}\left(\lambda_{1}+a_{1} \lambda_{2}\right)^{2}-4 \frac{\partial^{2} w}{\partial H \partial|A|^{2}} \frac{\partial F}{\partial \lambda_{1}}\left(1+a_{1}\right)\left(\lambda_{1}+a_{1} \lambda_{2}\right) .
\end{aligned}
$$

This formulae allow to easily compute evolution equations in critical points. 


\section{Other Normal Velocities}

10.1. Homogeneity less than minus one. In this section, we prove

Theorem 10.1. For any smooth closed strictly convex surface $M$ in $\mathbb{R}^{3}$, there exists a smooth family of surfaces $M_{t}, t \in[0, T)$, solving one of the following flow equations

- $\frac{d}{d t} X=\frac{H^{2}}{K^{2}} \nu$

- $\frac{d}{d t} X=\frac{|A|^{2}}{K^{2}} \nu$

- $\frac{d}{d t} X=\frac{H^{3}}{K^{3}} \nu$

with $M_{0}=M$. For $t \uparrow T, M_{t}$ converges to infinity. The rescaled surfaces $M_{t} \cdot r^{-1}(t)$ converge smoothly to the unit sphere $\mathbb{S}^{2}$, where $r(t)$ is the radius of an expanding sphere that converges to infinity for $t \uparrow T$, more precisely, $r(t)$ is as follows

- $\frac{d}{d t} X=\frac{H^{2}}{K^{2}} \nu: \quad r(t)=(4(T-t))^{-1}$,

- $\frac{d}{d t} X=\frac{|A|^{2}}{K^{2}} \nu: \quad r(t)=(2(T-t))^{-1}$,

- $\frac{d}{d t} X=\frac{H^{3}}{K^{3}} \nu: \quad r(t)=(16(T-t))^{-1 / 2}$.

We have also studied convex surfaces contracting according to

$$
\frac{d}{d t} X=-F \nu
$$

where $F$ is positive homogeneous of some degree larger than or equal to two [21]. There, we got the impression, that appropriate monotone quantities that assure convergence to a sphere after rescaling are available for almost every normal velocity considered. In contrast to this, such monotone quantities seem to be rare objects for expanding surfaces with normal velocity of homogeneity less than or equal to minus two.

In both cases, we restricted our attention to normal velocities and possible candidates for monotone quantities in $\mathbb{Z}\left(\lambda_{1}, \lambda_{2}\right)$ with small coefficients which are symmetric in $\lambda_{1}$ and $\lambda_{2}$.

In the case of expanding surfaces, however, we also find monotone quantities for surfaces expanding with a normal velocity that is positive homogeneous of degree minus one in the principal curvatures.

The proof of Theorem 10.1 is similar to the proof of Theorem 1.1. Therefore, we will present in the following only those results that are not almost identical to their counterparts in the proof of Theorem 1.1.

Theorem 10.2. For a family $M_{t}$ of smooth closed strictly convex surfaces in $\mathbb{R}^{3}$, flowing according to $\frac{d}{d t} X=\frac{H^{2}}{K^{2}} \nu$,

$$
\max _{M_{t}} \frac{\left(\lambda_{1}-\lambda_{2}\right)^{2}}{2\left(\lambda_{1}+\lambda_{2}\right) \lambda_{1} \lambda_{2}} \equiv \max _{M_{t}} w
$$

is non-increasing in time. 
Proof. According to Section 9, we obtain in a critical point of $w$

$$
\begin{aligned}
\frac{d}{d t} w-F^{i j} w_{; i j}= & -\frac{5\left(\lambda_{1}-\lambda_{2}\right)^{2}}{\lambda_{1}^{2} \lambda_{2}^{2}} \\
& -\frac{128}{\left(\lambda_{1}+3 \lambda_{2}\right)^{2} \lambda_{1}^{4}} \cdot h_{11 ; 1}^{2}+(\ldots) \cdot h_{22 ; 2}^{2}
\end{aligned}
$$

and apply the maximum principle.

The factor 2 in the denominator is useful to rewrite $w$ in terms of the algebraic basis consisting of $H$ and $|A|^{2}$

$$
\frac{\left(\lambda_{1}-\lambda_{2}\right)^{2}}{2\left(\lambda_{1}+\lambda_{2}\right) \lambda_{1} \lambda_{2}}=\frac{2|A|^{2}-H^{2}}{H \cdot\left(H^{2}-|A|^{2}\right)} .
$$

Theorem 10.3. For a family $M_{t}$ of smooth closed strictly convex surfaces in $\mathbb{R}^{3}$, flowing according to $\frac{d}{d t} X=\frac{|A|^{2}}{K^{2}} \nu$,

$$
\max _{M_{t}} \frac{\left(\lambda_{1}-\lambda_{2}\right)^{2}}{2\left(\lambda_{1}+\lambda_{2}\right) \lambda_{1} \lambda_{2}} \equiv \max _{M_{t}} w
$$

is non-increasing in time.

Proof. According to Section 9, we obtain in a critical point of $w$

$$
\begin{aligned}
\frac{d}{d t} w-F^{i j} w_{; i j}= & -\frac{2\left(2 \lambda_{1}^{2}+\lambda_{1} \lambda_{2}+2 \lambda_{2}^{2}\right)\left(\lambda_{1}-\lambda_{2}\right)^{2}}{\left(\lambda_{1}+\lambda_{2}\right)^{2} \lambda_{1}^{2} \lambda_{2}^{2}} \\
& -\frac{4\left(21 \lambda_{1}^{4}+24 \lambda_{1}^{3} \lambda_{2}+18 \lambda_{1}^{2} \lambda_{2}^{2}+\lambda_{2}^{4}\right)}{\left(\lambda_{1}+3 \lambda_{2}\right)^{2}\left(\lambda_{1}+\lambda_{2}\right)^{2} \lambda_{1}^{6}} \cdot h_{11 ; 1}^{2}+(\ldots) \cdot h_{22 ; 2}^{2}
\end{aligned}
$$

and apply the maximum principle.

Theorem 10.4. For a family $M_{t}$ of smooth closed strictly convex surfaces in $\mathbb{R}^{3}$, flowing according to $\frac{d}{d t} X=\frac{H^{3}}{K^{3}} \nu$,

$$
\max _{M_{t}} \frac{\left(\lambda_{1}+\lambda_{2}\right)^{6}\left(\lambda_{1}-\lambda_{2}\right)^{2}}{16\left(\lambda_{1}^{2}+\lambda_{2}^{2}\right)\left(\lambda_{1}^{2}+\lambda_{1} \lambda_{2}+\lambda_{2}^{2}\right) \lambda_{1}^{3} \lambda_{2}^{3}} \equiv \max _{M_{t}} w
$$

is non-increasing in time. 
Proof. According to Section 9, we obtain in a critical point of $w$

$$
\begin{aligned}
\frac{d}{d t} w-F^{i j} w_{; i j}=- & \frac{\left(\lambda_{1}+\lambda_{2}\right)^{8}\left(\lambda_{1}-\lambda_{2}\right)^{2}}{4\left(\lambda_{1}^{2}+\lambda_{2}^{2}\right)^{2}\left(\lambda_{1}^{2}+\lambda_{1} \lambda_{2}+\lambda_{2}^{2}\right)^{2} \lambda_{1}^{6} \lambda_{2}^{6}} . \\
& \cdot\left(\lambda_{1}^{6}-\lambda_{1}^{5} \lambda_{2}+8 \lambda_{1}^{4} \lambda_{2}^{2}+2 \lambda_{1}^{3} \lambda_{2}^{3}+8 \lambda_{1}^{2} \lambda_{2}^{4}-\lambda_{1} \lambda_{2}^{5}+\lambda_{2}^{6}\right) \\
- & \frac{3\left(\lambda_{1}+\lambda_{2}\right)^{8}}{8\left(3 \lambda_{1}^{6}+11 \lambda_{1}^{4} \lambda_{2}^{2}+2 \lambda_{1}^{3} \lambda_{2}^{3}+9 \lambda_{1}^{2} \lambda_{2}^{4}-2 \lambda_{1} \lambda_{2}^{5}+\lambda_{2}^{6}\right)^{2}} . \\
& \cdot \frac{1}{\left(\lambda_{1}^{2}+\lambda_{1} \lambda_{2}+\lambda_{2}^{2}\right)^{2}\left(\lambda_{1}^{2}+\lambda_{2}^{2}\right)^{2} \lambda_{1}^{8} \lambda_{2}^{6}} \cdot \\
& \cdot\left(2 \lambda_{1}^{18}-4 \lambda_{1}^{17} \lambda_{2}+57 \lambda_{1}^{16} \lambda_{2}^{2}-108 \lambda_{1}^{15} \lambda_{2}^{3}+508 \lambda_{1}^{14} \lambda_{2}^{4}\right. \\
& -428 \lambda_{1}^{13} \lambda_{2}^{5}+2152 \lambda_{1}^{12} \lambda_{2}^{6}-156 \lambda_{1}^{11} \lambda_{2}^{7}+4784 \lambda_{1}^{10} \lambda_{2}^{8} \\
& +172 \lambda_{1}^{9} \lambda_{2}^{9}+4942 \lambda_{1}^{8} \lambda_{2}^{10}-612 \lambda_{1}^{7} \lambda_{2}^{11}+2676 \lambda_{1}^{6} \lambda_{2}^{12} \\
& -772 \lambda_{1}^{5} \lambda_{2}^{13}+872 \lambda_{1}^{4} \lambda_{2}^{14}-340 \lambda_{1}^{3} \lambda_{2}^{15}+126 \lambda_{1}^{2} \lambda_{2}^{16} \\
& \left.-56 \lambda_{1} \lambda_{2}^{17}+9 \lambda_{2}^{18}\right) \cdot h_{11 ; 1}^{2} \\
+ & (\ldots) \cdot h_{22 ; 2}^{2} .
\end{aligned}
$$

Here and in the following, we write (...) to denote a term that equals the factor in front of $h_{11 ; 1}^{2}$ with $\lambda_{1}$ and $\lambda_{2}$ interchanged.

We finally apply the maximum principle.

Similarly as above, we obtain the following evolution equations for a family $M_{t}$ of surfaces flowing according to $\frac{d}{d t} X=-F \nu$

$$
\begin{aligned}
\frac{d}{d t} X^{\alpha}-F^{i j} X_{; i j}^{\alpha} & =\left(F^{i j} h_{i j}-F\right) \nu^{\alpha}, \\
\frac{d}{d t}|X|^{2}-F^{i j}\left(|X|^{2}\right)_{; i j} & =2\left(F^{i j} h_{i j}-F\right)\langle X, \nu\rangle-2 F^{i j} g_{i j}, \\
\frac{d}{d t} \nu^{\alpha}-F^{i j}\left(\nu^{\alpha}\right)_{; i j} & =F^{i j} h_{i}^{k} h_{k j} \cdot \nu^{\alpha}, \\
\frac{d}{d t}\langle X, \nu\rangle-F^{i j}\langle X, \nu\rangle_{; i j} & =-F^{i j} h_{i j}-F+F^{i j} h_{i}^{k} h_{k j}\langle X, \nu\rangle, \\
\frac{d}{d t} F-F^{i j} F_{; i j} & =F F^{i j} h_{i}^{k} h_{k j}, \\
\frac{d}{d t} g_{i j} & =-2 F h_{i j}, \\
\frac{d}{d t} h_{i j}-F^{k l} h_{i j ; k l} & =F^{k l} h_{k}^{a} h_{a l} \cdot h_{i j}-F^{k l} h_{k l} \cdot h_{i}^{a} h_{a j} \\
& -F h_{i}^{k} h_{k j}+F^{k l, r s} h_{k l ; i} h_{r s ; j} .
\end{aligned}
$$

Lemma 10.5. Under the assumptions of Theorem 10.1, $\frac{d}{d t} X=-F \nu$, the maximum principal curvature of $M_{t}$ is non-increasing in time.

Proof. Consider $M_{i j}:=h_{i j}-\mu g_{i j}$, where $\mu$ is chosen such that $M_{i j} \leq 0$ initially. We compute

$$
\begin{aligned}
\frac{d}{d t} M_{i j}-F^{k l} M_{i j ; k l}= & F^{k l} h_{k}^{a} h_{a l} \cdot h_{i j}-F^{k l} h_{k l} \cdot h_{i}^{a} h_{a j}-F h_{i}^{k} h_{k j}+2 \mu F h_{i j} \\
& +F^{k l, r s} h_{k l ; i} \xi^{i} h_{r s ; j} \xi^{j} .
\end{aligned}
$$


Assume that $\xi$ is a zero eigenvalue of $\left(M_{i j}\right), h_{i j} \xi^{j}=\mu g_{i j} \xi^{j}$, with $g_{i j} \xi^{i} \xi^{j}=1$. We may assume, that in our coordinate system, we have $\xi=(1,0)$ and $\left(h_{i j}\right)=\left(\begin{array}{ll}\mu & 0 \\ 0 & \lambda\end{array}\right)$ with $0<\lambda \leq \mu$. Normal velocities $F$ as in Theorem 10.1 are concave, so

$$
F^{k l, r s} h_{k l ; i} \xi^{i} h_{r s ; j} \xi^{j} \leq 0 \text {. }
$$

We estimate

- $\left(\frac{d}{d t} M_{i j}-F^{k l} M_{i j ; k l}\right) \xi^{i} \xi^{j} \leq-\frac{(\lambda+\mu)(3 \mu-\lambda)}{\lambda^{2}} \leq 0$ for $F=-\frac{H^{2}}{K^{2}}$,

- $\left(\frac{d}{d t} M_{i j}-F^{k l} M_{i j ; k l}\right) \xi^{i} \xi^{j} \leq-\frac{-2 \lambda \mu+\lambda^{2}+3 \mu^{2}}{\lambda^{2}} \leq 0$ for $F=-\frac{|A|^{2}}{K^{2}}$,

- $\left(\frac{d}{d t} M_{i j}-F^{k l} M_{i j ; k l}\right) \xi^{i} \xi^{j} \leq-\frac{2(\lambda+\mu)^{2}(2 \mu-\lambda)}{\mu \lambda^{3}} \leq 0$ for $F=-\frac{H^{3}}{K^{3}}$.

Now the claim follows directly from the maximum principle for tensors.

Lemma 10.6. Under the assumptions of Theorem 10.1, we obtain a lower bound on the principal curvatures similarly to Lemma 4.3.

Proof. We proceed as in the proof of Lemma 4.3. In a critical point of $\frac{-F}{2 R-\langle X, \nu\rangle}$, we compute the following evolution equation for a family of surfaces flowing according to $\frac{d}{d t} X=-F \nu$

$$
\begin{aligned}
\frac{d}{d t} \log \frac{-F}{2 R-\langle X, \nu\rangle}-F^{i j}\left(\log \frac{-F}{2 R-\langle X, \nu\rangle}\right)_{; i j}= & \frac{1}{2 R-\langle X, \nu\rangle} . \\
& \cdot\left(2 R \cdot F^{i j} h_{i}^{k} h_{k j}-F^{i j} h_{i j}-F\right) .
\end{aligned}
$$

We compute this explicitly for $F$ as in Theorem 10.1 and obtain in an increasing maximum of $\frac{-F}{2 R-\langle X, \nu\rangle}$

- $8 R-\frac{H}{K} \geq 0$ for $F=-\frac{H^{2}}{K^{2}}$,

- $4 H R-\frac{|A|^{2}}{K} \geq 0$ for $F=-\frac{|A|^{2}}{K}$,

- $6 R-\frac{H}{K} \geq 0$ for $F=-\frac{H^{3}}{K^{3}}$.

Our monotone quantities and Lemma 10.5 imply that our surfaces are pinched, i. e. that $\frac{\lambda_{1}}{\lambda_{2}}+\frac{\lambda_{2}}{\lambda_{1}}$ is uniformly bounded above. So we obtain there that $\lambda_{i} \geq \frac{1}{c R}$.

Lemma 10.7. Consider one of the flow equations of Theorem 10.1, $\frac{d}{d t} X=-F \nu$, and a solution as in this theorem. Let $\gamma$ be such that $F$ is positive homogeneous of degree $-\gamma$. Then there exists a constant $\alpha \gg 1$ such that $|F| \geq \frac{1}{\alpha}|X|^{\gamma}$ during the flow. More precisely, there exists $\alpha \gg 1$ such that $|X|^{\gamma}+\alpha F$ remains non-positive during the flow, if this quantity is negative initially.

Proof. For $F$ positive homogeneous of degree minus two, we get $F^{i j} h_{i j}=-2 F$ and obtain at a point, where $|X|^{2}+\alpha F=0$,

$$
\begin{aligned}
\frac{d}{d t}\left(|X|^{2}+\alpha F\right)-F^{i j}\left(|X|^{2}+\alpha F\right)_{; i j} & =-6 F\langle X, \nu\rangle-2 F^{i j} g_{i j}+\alpha F F^{i j} h_{i}^{k} h_{k j} \\
& \leq-6 F \sqrt{\alpha} \sqrt{-F}+\alpha F F^{i j} h_{i}^{k} h_{k j} .
\end{aligned}
$$

It is straightforward to check that for $\alpha \gg 1$ sufficiently large and $F$ as in the lemma, the right-hand side is non-positive.

If $F=-\frac{H^{3}}{K^{3}}$, we obtain similarly as above

$$
\frac{d}{d t}\left(|X|^{3}+\alpha F\right)-F^{i j}\left(|X|^{3}+\alpha F\right)_{; i j} \leq 12|X|^{2} \frac{H^{3}}{K^{3}}-6 \alpha \frac{H^{5}}{K^{5}} \leq 0 .
$$

In both cases, the lemma follows from the maximum principle. 


\begin{tabular}{|c||c|}
\hline$-\frac{1}{H}$ & $\frac{\left(\lambda_{1}-\lambda_{2}\right)^{2}}{\left(\lambda_{1}+\lambda_{2}\right) \lambda_{1} \lambda_{2}}$ \\
\hline$-\frac{1}{H}$ & $\frac{\left(\lambda_{1}^{2}+\lambda_{2}^{2}\right)\left(\lambda_{1}-\lambda_{2}\right)^{2}}{\left(\lambda_{1}+\lambda_{2}\right) \lambda_{1}^{3} \lambda_{2}^{3}}$ \\
\hline$-\frac{H}{K}$ & $\frac{\left(\lambda_{1}-\lambda_{2}\right)^{2}}{\lambda_{1}^{2} \lambda_{2}^{2}}$ \\
\hline
\end{tabular}

TABLE 2. More monotone quantities

10.2. Homogeneity minus one. In Table 2 , we have collected some normal velocities $F$, positive homogeneous of degree minus one, and test functions $w$, such that $\max _{M_{t}} w$ is non-increasing during the flow of a closed strictly convex surface flowing according to

$$
\frac{d}{d t} X=-F \nu
$$

Theorem 10.8. For a family $M_{t}$ of smooth closed strictly convex surfaces in $\mathbb{R}^{3}$, flowing according to $\frac{d}{d t} X=\frac{1}{H} \nu$,

$$
\max _{M_{t}} \frac{\left(\lambda_{1}-\lambda_{2}\right)^{2}}{2\left(\lambda_{1}+\lambda_{2}\right) \lambda_{1} \lambda_{2}} \equiv \max _{M_{t}} w
$$

and

are non-increasing in time.

$$
\max _{M_{t}} \frac{\left(\lambda_{1}^{2}+\lambda_{2}^{2}\right)\left(\lambda_{1}-\lambda_{2}\right)^{2}}{8\left(\lambda_{1}+\lambda_{2}\right) \lambda_{1}^{3} \lambda_{2}^{3}} \equiv \max _{M_{t}} \hat{w}
$$

Proof. According to Section 9, we obtain in a critical point of $w$

$$
\begin{aligned}
\frac{d}{d t} w-F^{i j} w_{; i j}= & -\frac{\left(\lambda_{1}^{2}+4 \lambda_{1} \lambda_{2}+\lambda_{2}^{2}\right)\left(\lambda_{1}-\lambda_{2}\right)^{2}}{2\left(\lambda_{1}+\lambda_{2}\right)^{3} \lambda_{1} \lambda_{2}} \\
& -\frac{2\left(5 \lambda_{1}^{2}+2 \lambda_{1} \lambda_{2}+\lambda_{2}^{2}\right) \lambda_{2}}{\left(\lambda_{1}+3 \lambda_{2}\right)^{2}\left(\lambda_{1}+\lambda_{2}\right) \lambda_{1}^{5}} \cdot h_{11 ; 1}^{2} \\
& +(\ldots) \cdot h_{22 ; 2}^{2}
\end{aligned}
$$

and apply the maximum principle.

Similarly, we obtain in a critical point of $\hat{w}$

$$
\begin{aligned}
\frac{d}{d t} \hat{w}-F^{i j} \hat{w}_{; i j}= & -\frac{\left(3 \lambda_{1}^{4}-2 \lambda_{1}^{2} \lambda_{2}^{2}+3 \lambda_{2}^{4}\right)\left(\lambda_{1}-\lambda_{2}\right)^{2}}{8\left(\lambda_{1}+\lambda_{2}\right)^{3} \lambda_{1}^{3} \lambda_{2}^{3}} \\
- & \frac{1}{2\left(3 \lambda_{1}^{3}+3 \lambda_{1}^{2} \lambda_{2}-\lambda_{1} \lambda_{2}^{2}+3 \lambda_{2}^{3}\right)^{2}\left(\lambda_{1}+\lambda_{2}\right)^{3} \lambda_{1}^{7} \lambda_{2}} . \\
& \cdot\left(9 \lambda_{1}^{10}-9 \lambda_{1}^{8} \lambda_{2}^{2}+96 \lambda_{1}^{7} \lambda_{2}^{3}-38 \lambda_{1}^{6} \lambda_{2}^{4}+96 \lambda_{1}^{5} \lambda_{2}^{5}+30 \lambda_{1}^{4} \lambda_{2}^{6}\right. \\
& \left.\quad+45 \lambda_{1}^{2} \lambda_{2}^{8}+27 \lambda_{2}^{10}\right) \cdot h_{11 ; 1}^{2} \\
& +(\ldots) \cdot h_{22 ; 2}^{2}
\end{aligned}
$$

and apply the maximum principle once again. 
Theorem 10.9. For a family $M_{t}$ of smooth closed strictly convex surfaces in $\mathbb{R}^{3}$, flowing according to $\frac{d}{d t} X=\frac{H}{K} \nu$,

$$
\max _{M_{t}} \frac{\left(\lambda_{1}-\lambda_{2}\right)^{2}}{4 \lambda_{1}^{2} \lambda_{2}^{2}} \equiv \max _{M_{t}} w
$$

is non-increasing in time.

Proof. According to Section 9, we obtain in a critical point of $w$

$$
\frac{d}{d t} w-F^{i j} w_{; i j}=-\frac{\left(\lambda_{1}-\lambda_{2}\right)^{2}}{\lambda_{1}^{2} \lambda_{2}^{2}}-\frac{2}{\lambda_{1}^{6}} \cdot h_{11 ; 1}^{2}+(\ldots) \cdot h_{22 ; 2} .
$$

and apply the maximum principle.

For these flow equations, convergence to infinity and convergence to a sphere after rescaling have been proved before for hypersurfaces $[11,26,29,30]$. Monotone quantities as mentioned above might at most be useful to improve the convergence rate. Inverse mean curvature was used to prove the Penrose inequality in general relativity [15]. Our techniques might also apply to surfaces expanding in the asymptotically flat manifolds considered there.

\section{Convergence Rate}

In order to find out what the optimal convergence rate might be, we proceed as in [21], use the same notation, and compute the linearized equation corresponding to

as

$$
\frac{d}{d t} X=\frac{1}{K} \nu-X \quad \text { or } \quad \frac{\partial u}{\partial t}=\frac{1}{K} w-u
$$

$$
\frac{\partial v}{\partial t}=\Delta v+v
$$

As in the contracting case, we only need to consider eigenvalues $-l(l+1)$ of the laplacian on the sphere for $l \in \mathbb{N}_{+}$. For $l=1$, we cannot expect convergence rates better than

$$
r_{+} \leq(T-t)^{-1} \cdot(1+c \cdot(T-t))
$$

if we fix $q(t)$ arbitrarily. This estimate is sharp, if we do not adjust $q(t)$. The corresponding eigenfunctions induce translations of the surface. Considering $l=2$, we expect that we cannot obtain convergence rates better than

$$
r_{+}(t) \leq(T-t)^{-1} \cdot\left(1+c \cdot(T-t)^{5}\right) .
$$

\section{REFERENCES}

[1] Ben Andrews, Fully nonlinear parabolic equations in two space variables, arXiv: math. AP/0402235.

[2] Ben Andrews, Moving surfaces by non-concave curvature functions, arXiv:math.DG/0402273.

[3] Ben Andrews, Contraction of convex hypersurfaces in Euclidean space, Calc. Var. Partial Differential Equations 2 (1994), no. 2, 151-171.

[4] Ben Andrews, Harnack inequalities for evolving hypersurfaces, Math. Z. 217 (1994), no. 2, 179-197.

[5] Ben Andrews, Gauss curvature flow: the fate of the rolling stones, Invent. Math. 138 (1999), no. $1,151-161$.

[6] Kai-Seng Chou and Xu-Jia Wang, A logarithmic Gauss curvature flow and the Minkowski problem, Ann. Inst. H. Poincaré Anal. Non Linéaire 17 (2000), no. 6, 733-751.

[7] Bennett Chow and Robert Gulliver, Aleksandrov reflection and nonlinear evolution equations. I. The n-sphere and n-ball, Calc. Var. Partial Differential Equations 4 (1996), no. 3, 249-264. 
[8] Bennett Chow and Peng Lu, The maximum principle for systems of parabolic equations subject to an avoidance set, Pacific J. Math. 214 (2004), no. 2, 201-222.

[9] Bennett Chow and Dong-Ho Tsai, Expansion of convex hypersurfaces by nonhomogeneous functions of curvature, Asian J. Math. 1 (1997), no. 4, 769-784.

[10] Bennett Chow and Dong-Ho Tsai, Nonhomogeneous Gauss curvature flows, Indiana Univ. Math. J. 47 (1998), no. 3, 965-994.

[11] Claus Gerhardt, Flow of nonconvex hypersurfaces into spheres, J. Differential Geom. 32 (1990), no. 1, 299-314.

[12] Claus Gerhardt, Closed Weingarten hypersurfaces in Riemannian manifolds, J. Differential Geom. 43 (1996), no. 3, 612-641.

[13] Richard S. Hamilton, Three-manifolds with positive Ricci curvature, J. Differential Geom. 17 (1982), no. 2, 255-306.

[14] Gerhard Huisken, Flow by mean curvature of convex surfaces into spheres, J. Differential Geom. 20 (1984), no. 1, 237-266.

[15] Gerhard Huisken and Tom Ilmanen, The inverse mean curvature flow and the Riemannian Penrose inequality, J. Differential Geom. 59 (2001), no. 3, 353-437.

[16] Gerhard Huisken and Tom Ilmanen, Higher regularity of the inverse mean curvature flow, 2002, http://www.math.ethz.ch/ ilmanen/papers/pub.html.

[17] Gerhard Huisken and Alexander Polden, Geometric evolution equations for hypersurfaces, Calculus of variations and geometric evolution problems (Cetraro, 1996), Lecture Notes in Math., vol. 1713, Springer, Berlin, 1999, pp. 45-84.

[18] Nina M. Ivochkina, Thomas Nehring, and Friedrich Tomi, Evolution of starshaped hypersurfaces by nonhomogeneous curvature functions, Algebra i Analiz 12 (2000), no. 1, 185-203.

[19] James A. McCoy, The surface area preserving mean curvature flow, Asian J. Math. 7 (2003), no. $1,7-30$.

[20] Oliver C. Schnürer, Translating solutions to the second boundary value problem for curvature flows, Manuscripta Math. 108 (2002), no. 3, 319-347.

[21] Oliver C. Schnürer, Surfaces contracting with speed $|A|^{2}$, J. Differential Geom. 71 (2005), no. 3, 347-363, arXiv:math.DG/0409388.

[22] Oliver C. Schnürer and Knut Smoczyk, Evolution of hypersurfaces in central force fields, J. Reine Angew. Math. 550 (2002), 77-95.

[23] Oliver C. Schnürer and Knut Smoczyk, Neumann and second boundary value problems for Hessian and Gauß curvature flows, Ann. Inst. H. Poincaré. Anal. Non Linéaire 20 (2003), no. 6, 1043-1073.

[24] Felix Schulze, appendix with Oliver C. Schnürer, Convexity estimates for flows of hypersurfaces by powers of the mean curvature, 2005, in preparation.

[25] Knut Smoczyk, Remarks on the inverse mean curvature flow, Asian J. Math. 4 (2000), no. 2, 331-335.

[26] Knut Smoczyk, A representation formula for the inverse harmonic mean curvature flow, 2003, MPI-MIS Preprint 85/2003, http://www.mis.mpg.de/.

[27] Michael Spivak, A comprehensive introduction to differential geometry. Vol. IV. 2nd ed., Berkeley: Publish Perish, Inc. VII, 561 p., 1979.

[28] Kaising Tso, Deforming a hypersurface by its Gauss-Kronecker curvature, Comm. Pure Appl. Math. 38 (1985), no. 6, 867-882.

[29] John I. E. Urbas, On the expansion of starshaped hypersurfaces by symmetric functions of their principal curvatures, Math. Z. 205 (1990), no. 3, 355-372.

[30] John I. E. Urbas, An expansion of convex hypersurfaces, J. Differential Geom. 33 (1991), no. $1,91-125$.

FU Berlin, Arnimallee 2-6, 14195 Berlin, Germany

E-mail address: Oliver.Schnuerer@math.fu-berlin.de 